JOURNAL OF

SYMPLECTIC GEOMETRY

Volume 12, Number 3, 553-583, 2014

\title{
BILINEARIZED LEGENDRIAN CONTACT HOMOLOGY AND THE AUGMENTATION CATEGORY
}

\author{
Frédéric Bourgeois and Baptiste Chantraine
}

In this paper, we construct an $\mathcal{A}_{\infty}$-category associated to a Legendrian submanifold of a jet space. Objects of the category are augmentations of the Chekanov algebra $\mathcal{A}(\Lambda)$ and the homology of the morphism spaces forms a new set of invariants of Legendrian submanifolds called the bilinearized Legendrian contact homology. Those are constructed as a generalization of linearized Legendrian contact homology using two augmentations instead of one. Considering similar constructions with more augmentations leads to the higher order composition maps in the category and generalizes the idea of $[6]$ where an $\mathcal{A}_{\infty}$-algebra was constructed from one augmentation. This category allows us to define a notion of equivalence of augmentations when the coefficient ring is a field regardless of its characteristic. We use simple examples to show that bilinearized cohomology groups are efficient to distinguish those equivalences classes. We also generalize the duality exact sequence from [12] in our context, and interpret geometrically the bilinearized homology in terms of the Floer homology of Lagrangian fillings (following $[8])$.

\section{Contents}

\section{Introduction}

2. Algebraic setup 
2.3. Equivalence of non-unital $\mathcal{A}_{\infty}$-categories $\quad 562$ 2.3.1. Yoneda modules $\quad 564$

2.3.2. Cohomological adjunctions $\quad 565$

2.4. Invariance $\quad 568$

2.4.1. Construction of functors on $\operatorname{Aug}(A) \quad 568$

2.4.2. Equivalence of augmentations $\quad 569$

2.4.3. Invariance under stable isomorphisms $\quad 569$

3. Geometric interpretation $\quad \mathbf{5 7 0}$

3.1. Legendrian contact homology 570

3.2. The augmentation category of Legendrian submanifolds

3.2.1. The augmentation category $\operatorname{Aug}(\Lambda) \quad 572$

3.2.2. The $n$-copy Legendrian link 572

3.2.3. Reeb chords of $\Lambda_{n} \quad 572$

3.2.4. The differential 573

3.2.5. Structure of $\partial_{n}$

3.3. Duality exact sequence $\quad 575$

4. Perspectives $\quad 577$

4.1. Lagrangian fillings $\quad 577$

4.2. Relation with generating families $\quad 578$

5. Examples $\quad \mathbf{5 7 8}$

5.1. Trefoil knot $\quad 579$

5.2. Chekanov-Eliashberg knot 581

References $\quad \mathbf{5 8 2}$

\section{Introduction}

For a Legendrian submanifold $\Lambda$ of a jet space $\mathcal{J}^{1}(M)$ Legendrian contact homology is the homology of a non-commutative differential algebra freely generated by double points of the projection of $\Lambda$ to $T^{*}(M)$ constructed in $[\mathbf{5}]$ for $M \simeq \mathbb{R}$ and in $[\mathbf{9 , 1 1}]$ in general. We refer to this algebra as the Chekanov algebra of $\Lambda$ denoted by $(\mathcal{A}(\Lambda), \partial)$ whose homology is denoted by $\mathrm{LCH}(\Lambda)$. The boundary operator counts holomorphic curves in the symplectization whose domains are disks with points removed on the boundary. At one of these points, the holomorphic curve is required to have one positive asymptotic and at all the others it is required to have a negative asymptotic. When non-zero, Legendrian contact homology is most of time infinite dimensional, hence this turns out to be difficult to distinguish two non-isomorphic LCH algebras. 
Linearization of semi-free differential graded algebras (DGA) is a process used in [5], which associates finite-dimensional invariants of Legendrian submanifolds from their Chekanov algebras. Linearization is done using augmentations of $\mathcal{A}(\Lambda)$ that is DGA-maps $\varepsilon: \mathcal{A}(\Lambda) \rightarrow \mathbb{K}$ where $\mathbb{K}$ is the coefficient field we consider (most frequently $\mathbb{Z}_{2}$ ). Those invariants have the advantage to be both computable (once the boundary operator of Legendrian contact homology is known) and efficient to distinguish Legendrian submanifolds which were not distinguished by other invariants. However, the process of linearization makes the theory commutative. Also, it is not efficient regarding the question of distinguishing augmentations of Chekanov algebra.

In this paper, we propose a new invariant called bilinearized Legendrian contact homology which closely follows the process of linearization but uses the fact that the theory is non-commutative in order to use two augmentations instead of one.

More precisely, for each pair of augmentations $\left(\varepsilon_{0}, \varepsilon_{1}\right)$ we define a differential

$$
d^{\varepsilon_{0}, \varepsilon_{1}}: C(\Lambda) \rightarrow C(\Lambda)
$$

in homology, and its dual

$$
\mu_{\varepsilon_{1}, \varepsilon_{0}}^{1}: C^{*}(\Lambda) \rightarrow C^{*}(\Lambda)
$$

in cohomology, where $C(\Lambda)$ is the vector space over $\mathbb{K}$ generated by Reeb chords of $\Lambda$. We denote by $C^{\varepsilon_{0}, \varepsilon_{1}}$ the complex $\left(C(\Lambda), d^{\varepsilon_{0}, \varepsilon_{1}}\right)$, and by $C_{\varepsilon_{1}, \varepsilon_{0}}$ the complex $\left(C^{*}(\Lambda), \mu_{\varepsilon_{1}, \varepsilon_{0}}^{1}\right)$. The homologies of those complexes are the bilinearized (co)homology groups, we denote them by $\mathrm{LCH}^{\varepsilon_{0}, \varepsilon_{1}}(\Lambda)$ and $\mathrm{LCH}_{\varepsilon_{1}, \varepsilon_{0}}(\Lambda)$, respectively. Those are generalizations of the standard linearized Legendrian contact homology, as it will appear obvious from the definition that the differential $d^{\varepsilon, \varepsilon}$ is the standard augmented differential $d^{\varepsilon}$ from $[5,9]$.

Our first result is that the set of those homologies is a Legendrian isotopy invariant as stated in the following

Theorem 1.1. The set of isomorphism classes of $\mathrm{LCH}_{\varepsilon_{1}, \varepsilon_{0}}(\Lambda)$ (or of $\left.\mathrm{LCH}^{\varepsilon_{0}, \varepsilon_{1}}(\Lambda)\right)$ over all pairs of augmentations $\left(\varepsilon_{0}, \varepsilon_{1}\right)$ of $\mathcal{A}(\Lambda)$ is a Legendrian isotopy invariant.

Geometrically, bilinearized Legendrian contact homology associated to two augmentations $\varepsilon_{0}$ and $\varepsilon_{1}$ amounts to counting the holomorphic curves decorated with the augmentations. An example of such a decorated curve is shown in Figure 1. Note that the number of negative asymptotics on the left and on the right could be arbitrary (see equation (2.5) for an explicit algebraic definition). 


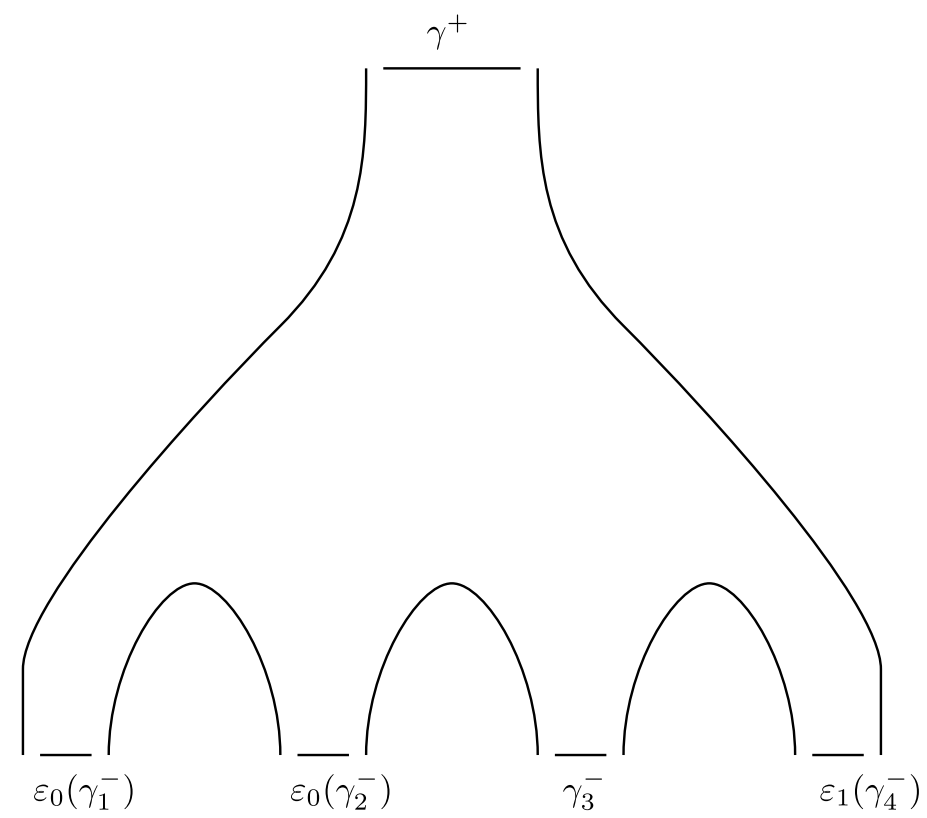

Figure 1. Curve contributing to $d^{\varepsilon_{0} ; \varepsilon_{1}}\left(\gamma^{+}\right)\left(\right.$or $\left.\mu_{\varepsilon_{0}, \varepsilon_{1}}^{1}\left(\gamma_{3}^{-}\right)\right)$.

We can use similar ideas and consider holomorphic curves decorated by $d+1 \geq 3$ augmentations to build structural maps $\mu_{\varepsilon_{d}, \ldots, \varepsilon_{0}}^{d}: C_{\varepsilon_{d}, \varepsilon_{d-1}} \otimes \cdots \otimes$ $C_{\varepsilon_{1}, \varepsilon_{0}} \rightarrow C_{\varepsilon_{d}, \varepsilon_{0}}$. Figure 2 gives an example of such a curve, again the number of decorated negative ends is in general arbitrary, equation (2.4) gives the explicit formula of this operation.

Those structural maps satisfy an $\mathcal{A}_{\infty}$-relation which allows us to define an $\mathcal{A}_{\infty}$-category whose objects are augmentations of the Chekanov algebra

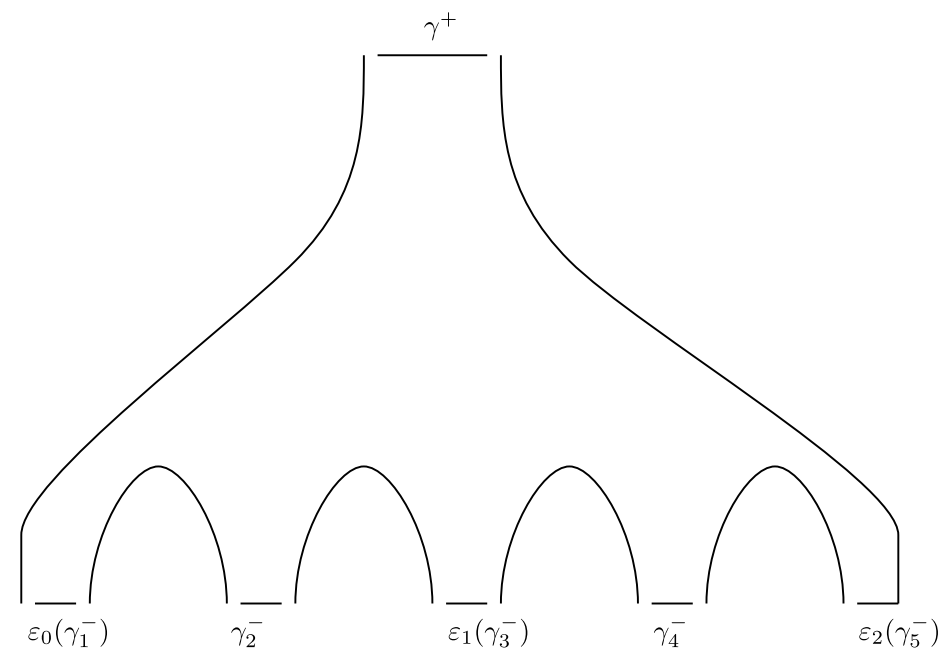

Figure 2. Curve contributing to $\left.\mu_{\varepsilon_{0}, \varepsilon_{1}, \varepsilon_{2}}^{2}\left(\gamma_{2}^{-}, \gamma_{4}^{-}\right)\right)$. 
of $\Lambda$. We call this category the augmentation category of $\Lambda$. We denote it by $\operatorname{Aug}(\Lambda)$. This is a direct generalization of the $\mathcal{A}_{\infty}$-algebra constructed in [6]. Under Legendrian isotopy this category changes by a pseudo-equivalence as stated in the following:

Theorem 1.2. If $\Lambda$ is Legendrian isotopic to $\Lambda^{\prime}$ then the categories $\operatorname{Aug}(\Lambda)$ and $\operatorname{Aug}\left(\Lambda^{\prime}\right)$ are pseudo-equivalent.

These categories are not (in general) cohomologically unital (in the sense of [17]). Therefore one cannot take the definition of quasi-equivalence as in [17]. In Section 2.3, we introduce the notion of pseudo-equivalence which will coincide with the notion of quasi-equivalence in the case of $c$-unital categories. We borrow many notations from $[\mathbf{1 7}]$ and try to give the necessary definitions to understand the main concepts in the present paper. As it will follow from this section that the morphism spaces in the corresponding homological categories are invariant under quasi-equivalences, Theorem 1.1 is a corollary of Theorem 1.2.

The benefit of having a category where the objects are augmentations of $\mathcal{A}(\Lambda)$ is that it allows us to define the notion of equivalence of augmentations even when the ground field $\mathbb{K}$ is not of characteristic 0 (compare with the definition in $[\mathbf{3}]$ ). After precisely defining equivalence of augmentations in Section 2.4.2 we prove that

Theorem 1.3. If $\Lambda$ is Legendrian isotopic to $\Lambda^{\prime}$ then the quasi-equivalence of Theorem 1.2 induces a bijection between the equivalence classes of augmentation of $\mathcal{A}(\Lambda)$ and those of $\mathcal{A}\left(\Lambda^{\prime}\right)$.

In particular, if the characteristic of $\mathbb{K}$ is finite one gets that the (finite) number of equivalence classes is a Legendrian invariant.

The group $\mathrm{LCH}^{\varepsilon_{0}, \varepsilon_{1}}$ appears to be an efficient tool to distinguish some of those equivalence classes of augmentations using the following theorem.

Theorem 1.4. If $\varepsilon_{1}$ and $\varepsilon_{2}$ are equivalent then for all augmentations $\varepsilon$

$$
\operatorname{LCH}^{\varepsilon_{1}, \varepsilon}(\Lambda) \simeq \operatorname{LCH}^{\varepsilon_{2}, \varepsilon}(\Lambda)
$$

and

$$
\mathrm{LCH}^{\varepsilon, \varepsilon_{1}}(\Lambda) \simeq \mathrm{LCH}^{\varepsilon, \varepsilon_{2}}(\Lambda) .
$$

The first part of the paper (Section 2) is devoted to the algebraic construction of the augmentation category for any semi-free DGA, we borrow the necessary definitions regarding $\mathcal{A}_{\infty}$-categories from $[\mathbf{1 7}]$ and try to make this paper as self-contained as possible. In the second part, we apply this algebraic construction to the case of the Chekanov algebra of a Legendrian submanifold. We also give a geometrical interpretation of the bilinearized differential in terms of the Chekanov algebra of the 2-copy Legendrian link. We also investigate in Section 3.3 the generalization of the duality exact sequence from $[\mathbf{1 2}]$, and we prove the following theorem. 
Theorem 1.5. Let $\varepsilon_{0}$ and $\varepsilon_{1}$ be two augmentations of $\Lambda$ where $\Lambda \subset \mathcal{J}^{1}(M)$ is horizontally displaceable. Then there is a long exact sequence

$$
\cdots \rightarrow H_{k+1}(\Lambda) \stackrel{\sigma^{\varepsilon_{0}, \varepsilon_{1}}}{\longrightarrow} \mathrm{LCH}_{\varepsilon_{1}, \varepsilon_{0}}^{n-k-1}(\Lambda) \rightarrow \mathrm{LCH}_{k}^{\varepsilon_{0}, \varepsilon_{1}}(\Lambda) \stackrel{\rho^{\varepsilon_{0}, \varepsilon_{1}}}{\longrightarrow} H_{k}(\Lambda) \rightarrow \cdots
$$

In Section 4.1, we interpret the bilinearized Legendrian contact homology when $\varepsilon_{1}$ and $\varepsilon_{2}$ come from Lagrangian fillings in terms of the Lagrangian Floer homology of those fillings (as defined in [8]). In Section 5, we provide some simple examples of computations which demonstrate the effectiveness of Theorem 1.4 to distinguish augmentations.

\section{Algebraic setup}

\subsection{The $n$-copy algebra of a free DGA.}

2.1.1. The $n$-copy algebra. Let $\mathbb{K}$ be a field. For each $n \in \mathbb{N}$ we denote by $\mathbb{K}_{n}$ the semi-simple algebra over $\mathbb{K}$ generated by $e_{i}, i \in\{1, \ldots, n\}$, with the relations $e_{i} \cdot e_{j}=\delta_{i, j} e_{i}$ and $\sum e_{i}=1$. For a set $A$ we denote by $C_{n}(A)$ the free $\mathbb{K}_{n}$-bimodule generated by the elements of $A$ and by

$$
\mathcal{A}_{n}(A)=\mathbb{K}_{n} \oplus C_{n}(A) \oplus\left(C_{n}(A) \otimes C_{n}(A)\right) \oplus \cdots \oplus C_{n}(A)^{\otimes k} \oplus \cdots
$$

the tensor algebra over $\mathbb{K}_{n}$ of $C_{n}(A)$ (here $\otimes$ denotes the tensor product of $\mathbb{K}_{n}$-modules). For each $a \in A$ we denote by $a_{i, j}$ the element $e_{i} \cdot a \cdot e_{j} \in C_{n}(A)$. Note that as a $\mathbb{K}_{n}$-bimodule $C_{n}(A)$ decomposes as $\bigoplus_{(i, j)} C_{n}(A)_{i, j}$ where $C_{n}(A)_{i, j}$ is the submodule generated by the $a_{i, j}$. In the tensor algebra $\mathcal{A}_{n}(A)$, $C_{n}(A)_{i, j} \otimes C_{n}(A)_{k, l}$ is non-zero iff $j=k$. A pair of multi-indices $(I, J)$ of the same length $k$, i.e., $I=\left(i_{1}, \ldots, i_{k}\right)$ and $J=\left(j_{1}, \ldots, j_{k}\right)$, is said to be composable if for every $l=1, \ldots, k-1$ we have $j_{l}=i_{l+1}$. Then the tensor product $C_{n}(A)_{I, J}:=C_{n}(A)_{i_{1}, j_{1}} \otimes \cdots \otimes C_{n}(A)_{i_{k}, j_{k}}$ does not vanish iff $(I, J)$ is composable, so that the tensor algebra $\mathcal{A}_{n}(A)$ decomposes as a $\mathbb{K}_{n}$-bimodule as the direct sum of submodules $C_{n}(A)_{I, J}$ over all composable pairs $(I, J)$. Note that, for $i=1, \ldots, n$, the $\mathbb{K}$-subalgebra $\mathcal{A}_{n}(A)_{i} \subset \mathcal{A}_{n}(A)$ defined as the tensor algebra of $C_{n}(A)_{i, i}$, and corresponding to multi-indices $I$ and $J$ having all components equal to $i$, is naturally isomorphic to $\mathcal{A}(A):=\mathcal{A}_{1}(A)$ as a $\mathbb{K}$-algebra.

Now suppose that the tensor algebra $\mathcal{A}(A)$ is equipped with a differential $\partial$ such that $(\mathcal{A}(A), \partial)$ is a differential algebra. We define a differential $\partial_{n}$ on $\mathcal{A}_{n}(A)$ by setting $\partial_{n}\left(e_{i} \cdot a \cdot e_{j}\right)=e_{i} \cdot \partial(a) \cdot e_{j}$ for all $a \in A$, where $\partial(a)$ is interpreted as an element of $\mathcal{A}_{n}(A)$. We then extend $\partial_{n}$ to $\mathcal{A}_{n}(A)$ by 
linearity and by the Leibniz rule. Note that since $a=\sum_{i, j} a_{i, j}$ in $\mathcal{A}_{n}(A)$ and $\sum_{i} e_{i}=1$ in $\mathbb{K}_{n}$ the formula tells us that any word of length $k$ in $\partial(a)$ appears as a sum in $\partial_{n}(a)$ over all composable pairs of multi-indices of length $k$.

2.1.2. Grading. Assume also that the set $A$ comes with a grading map gr : $A \rightarrow \mathbb{Z}$ which defines a grading on homogeneous elements of $\mathcal{A}(A)$ by $\operatorname{gr}(a b)=\operatorname{gr}(a)+\operatorname{gr}(b)$. Defining the grading of elements of $\mathbb{K}_{n}$ to be 0 , this extends to a grading on $\mathcal{A}_{n}(A)$. From now on we assume that the differential $\partial$ has degree -1 , so that $\partial_{n}$ has degree -1 as well and turns $\left(\mathcal{A}_{n}(A), \partial_{n}\right)$ a DGA over $\mathbb{K}_{n}$. This means that the Leibniz rule becomes: $\partial(a b)=\partial(a) \cdot b+(-1)^{\operatorname{gr}(a)} a \cdot \partial(b)$. The DGA $(\mathcal{A}, \partial)$ with generating set $A$ is commonly referred in the literature to a semi-free DGA.

In order to agree with the degree convention in the literature (see [17]), there is a stabilization process when going from the language of DGAs to the language of $\mathcal{A}_{\infty}$-categories which induces a difference between the notions of grading. We will give two different notations for those two different gradings. We denote by $\operatorname{gr}(a)$ the grading of $a \in A$ as a generator of $\mathcal{A}$, this grading will be referred to as the reduced grading according to the literature. We will denote by $|a|=\operatorname{gr}(a)+1$ the absolute grading of $a$ as a generator of the dual module $C^{*}(A)$. Although we will pay careful attention to our notations, the convention is that whenever we speak in the world of $\mathcal{A}_{\infty}$-categories the grading is assumed to be the absolute grading and in the world of DGA the grading is assumed to be the reduced grading.

If $V$ is a graded vector space, we denote by $V[d]$ the graded vector space isomorphic to $V$ with degree shifted by $d$ (i.e., the grading of $a$ in $V[d]$ is $\operatorname{gr}(a)-d)$. When defined between graded vector space, we assume that all our linear maps preserve the degree hence a map $F: V \rightarrow W$ shifting the degree by $d$ will be denoted by $F: V \rightarrow W[d]$.

If $T:\left(V, \mu_{V}^{1}\right) \rightarrow\left(W[d], \mu_{W}^{1}\right)$ is a linear map between chain complexes, we will sometimes denote for short $T \circ \mu_{V}^{1}+(-1)^{d} \mu_{W}^{1} \circ T$ by $\mu^{1}(T)$.

2.1.3. Augmentations of $\mathcal{A}_{\boldsymbol{n}}(\boldsymbol{A})$. Recall that an augmentation of a DGA $\mathcal{A}$ over $\mathbb{K}$ is a DGA map from $(\mathcal{A}, \partial)$ to $(\mathbb{K}, 0)$. More precisely it as a map $\varepsilon: \mathcal{A} \rightarrow \mathbb{K}$ satisfying

- $\varepsilon(a)=0$ if $\operatorname{gr}(a) \neq 0$,

- $\varepsilon \circ \partial=0$,

- $\varepsilon(a b)=\varepsilon(a) \cdot \varepsilon(b)$.

Let $E=\left(\varepsilon_{1}, \ldots, \varepsilon_{n}\right)$ be a $n$-tuple of augmentations of $\mathcal{A}(A)$ over $\mathbb{K}$. We define $\varepsilon_{E}: \mathcal{A}_{n}(A) \rightarrow \mathbb{K}_{n}$ by $\varepsilon_{E}(a)=\sum_{i} \varepsilon_{i}\left(e_{i} \cdot a \cdot e_{i}\right) \cdot e_{i}$. In other words, $\varepsilon_{E}$ equals $\varepsilon_{i} \cdot e_{i}$ on $\mathcal{A}(A)_{i}$, and $\varepsilon_{E}$ vanishes on $C_{n}(A)_{I, J}$ when the multi-indices $I$ and $J$ are not constant.

Proposition 2.1. $\varepsilon_{E}$ is an augmentation of $\mathcal{A}_{n}(A)$ over $\mathbb{K}_{n}$. 
Proof. First, one must check that $\varepsilon_{E}$ is an algebra morphism. For $a \in$ $C_{n}(A)_{I, J}$ and $b \in C_{n}(A)_{K, L}$, we have $\varepsilon_{E}(a \cdot b)=\varepsilon_{i}(a \cdot b) \cdot e_{i}=\varepsilon_{i}(a) \varepsilon_{i}(b) \cdot e_{i}=$ $\varepsilon_{E}(a) \varepsilon_{E}(b)$ if $I, J, K, L$ have all components equal to $i$. Otherwise, $a \cdot b=0$ so that $\varepsilon_{E}(a \cdot b)=0$ and at least one of $\varepsilon_{E}(a)$ or $\varepsilon_{E}(b)$ vanishes as well.

Second, one must check that $\varepsilon_{E} \circ \partial_{n}=0$. Since $\partial_{n}\left(e_{i} \cdot a \cdot e_{j}\right)=e_{i} \cdot \partial(a) \cdot e_{j}$, we have $\varepsilon_{E} \circ \partial_{n}\left(e_{i} \cdot a \cdot e_{j}\right)=e_{i} \cdot \varepsilon_{i} \circ \partial(a) \delta_{i, j}$ which vanishes since $\varepsilon_{i}$ is an augmentation.

Using the augmentation $\varepsilon_{E}$ one defines the $\mathbb{K}_{n}$-algebra map $\phi_{\varepsilon_{E}}: \mathcal{A}_{n} \rightarrow$ $\mathcal{A}_{n}$ which sends a generator $a$ to $a+\varepsilon_{E}(a)$. Consider the induced differential $\partial^{\varepsilon_{E}}=\phi_{\varepsilon_{E}} \circ \partial_{n} \circ \phi_{\varepsilon_{E}}^{-1}$. Since $\varepsilon_{E}$ is an augmentation, $\left.\partial^{\varepsilon_{E}}\right|_{C_{n}(A)}$ has no constant term and hence decomposes as a sum $\partial_{1}^{\varepsilon_{E}} \oplus \partial_{2}^{\varepsilon_{E}} \oplus \cdots \oplus \partial_{d}^{\varepsilon_{E}} \oplus \cdots$ where $\partial_{d}^{\varepsilon_{E}}: C_{n}(A) \rightarrow C_{n}(A)^{\otimes d}[-1]$. From $\partial^{\varepsilon_{E}} \circ \partial^{\varepsilon_{E}}=0$ one gets that $\partial_{1}^{\varepsilon_{E}} \circ \partial_{1}^{\varepsilon_{E}}=0$.

Dualizing each of $\partial_{d}^{\varepsilon_{E}}$ one gets a family of maps $\mu_{\varepsilon_{E}}^{d, n}: C_{n}^{*}(A)^{\otimes d} \rightarrow$ $C_{n}^{*}(A)[2-d]$ (using the absolute grading here) defined by the relation:

$$
\mu^{d, n}(f)(b)=f\left(\partial_{d}^{\varepsilon_{E}}(b)\right)
$$

for any $\mathbb{K}_{n}$-balanced form $f$ on $C_{n}(A)$.

Similarly to $[\mathbf{6}]$ from $\partial^{\varepsilon_{E}} \circ \partial^{\varepsilon_{E}}=0$ one gets that the family of maps $\mu_{\varepsilon_{E}}^{d, n}$ is an $\mathcal{A}_{n}$-algebra structure on $C_{n}^{*}(A)$ that is:

$$
\begin{aligned}
& \sum_{i=1}^{d} \sum_{j=0}^{d-i}(-1)^{\mathbf{H}_{j}} \mu_{\varepsilon_{E}}^{d-i+1, n}\left(a_{d}, a_{d-1}, \ldots, a_{j+i+1}, \mu_{\varepsilon_{E}}^{i, n}\left(a_{j+i}, \ldots, a_{j+1}\right), a_{j}, \ldots, a_{1}\right) \\
& \quad=0
\end{aligned}
$$

where $\boldsymbol{\Psi}_{j}=\left|a_{1}\right|+\cdots+\left|a_{j}\right|-j$.

\subsection{The augmentation category.}

2.2.1. Bilinearized complexes. Note that $\mu_{\varepsilon_{E}}^{d, n}$ maps $C_{i_{d}, i_{d-1}}^{*} \otimes C_{i_{d-1}, i_{d-2}}^{*} \otimes$ $\cdots \otimes C_{i_{1}, i_{0}}^{*}$ to $C_{i_{d}, i_{0}}^{*}[2-d]$ and that this map depends only on $\partial$ and $\varepsilon_{i_{d}}, \varepsilon_{i_{d-1}}, \ldots, \varepsilon_{i_{0}}$. This implies that, after identifying each $C_{i, j}^{*}$ with $C^{*}(A)$ for each $(d+1)$-tuple of augmentations $\varepsilon_{0}, \ldots, \varepsilon_{d}$ the map $\mu_{\varepsilon_{E}}^{d, n}$ induces a map $\mu_{\varepsilon_{d}, \ldots, \varepsilon_{0}}^{d}: C^{*}(A)^{\otimes d} \rightarrow C^{*}(A)[2-d]$ independent of $n \geq d+1$ and the remaining augmentations in $E$. A similar discussion holds for the map $\partial_{d}^{\varepsilon_{E}}: C(A) \rightarrow C(A)[-1]$. From equation (2.1) one deduces that:

$$
\begin{aligned}
& \sum_{i=1}^{d} \sum_{j=0}^{d-i}(-1)^{\mathbf{z}_{j}} \mu_{\varepsilon_{d}, \ldots, \varepsilon_{j+i}, \varepsilon_{j}, \ldots, \varepsilon_{0}}^{d-i+1}\left(a_{d}, a_{d-1}, \ldots, a_{j+i+1}, \mu_{\varepsilon_{j+i}, \ldots, \varepsilon_{j}}^{i}\left(a_{j+i}, \ldots,\right.\right. \\
& \left.\left.\quad a_{j+1}\right), a_{j}, \ldots, a_{1}\right)=0 .
\end{aligned}
$$


In particular, when $d=1$ one gets $\mu_{\varepsilon_{1}, \varepsilon_{0}}^{1} \circ \mu_{\varepsilon_{1}, \varepsilon_{0}}^{1}=0$, similarly defining $d^{\varepsilon_{0}, \varepsilon_{1}}$ the map on $C(A)$ associated to $\partial_{1}^{\varepsilon_{E}}$ one gets $d^{\varepsilon_{0}, \varepsilon_{1}} \circ d^{\varepsilon_{0}, \varepsilon_{1}}=0$. Hence, we get two complexes called the bilinearized complexes, $C_{\varepsilon_{1}, \varepsilon_{0}}=\left(C^{*}(A), \mu_{\varepsilon_{1}, \varepsilon_{0}}^{1}\right)$ and $C^{\varepsilon_{0}, \varepsilon_{1}}=\left(C(A), d^{\varepsilon_{0}, \varepsilon_{1}}\right)$. The homology of $C^{\varepsilon_{0}, \varepsilon_{1}}$ is called the bilinearized homology of $(\mathcal{A}(A), \partial)$ with respect to $\varepsilon_{0}$ and $\varepsilon_{1}$ and the homology of $C_{\varepsilon_{1}, \varepsilon_{0}}$ is called the bilinearized cohomology.

The maps $\mu_{\varepsilon_{d}, \ldots, \varepsilon_{0}}^{d}$ are higher order compositions of an $\mathcal{A}_{\infty}$-category we describe here.

2.2.2. Definition of $\operatorname{Aug}(\boldsymbol{A})$. The augmentation category $\operatorname{Aug}(A)$ is the $\mathcal{A}_{\infty}$-category defined by

- $\mathrm{Ob}(\operatorname{Aug}(A))$ is the set of augmentations of $\mathcal{A}$,

- Morphisms from $\varepsilon_{1}$ to $\varepsilon_{2}$ are the complexes $C_{\varepsilon_{2}, \varepsilon_{1}}=\left(C^{*}(A), \mu_{\varepsilon_{2}, \varepsilon_{1}}^{1}\right)$,

- The $\mathcal{A}_{\infty}$-composition maps are

$$
\mu_{\varepsilon_{d}, \ldots, \varepsilon_{0}}^{d}: C_{\varepsilon_{d}, \varepsilon_{d-1}} \otimes \cdots \otimes C_{\varepsilon_{1}, \varepsilon_{0}} \rightarrow C_{\varepsilon_{d}, \varepsilon_{0}}[2-d]
$$

In order to facilitate computations, we unravel here the construction to give an explicit formula for those maps. If $\partial\left(a_{j}\right)=\sum_{i_{1}, \ldots, i_{k}} x_{i_{1}, \ldots, i_{k}}^{j} \cdot b_{i_{1}}^{j} \ldots b_{i_{k}}^{j}$ with $x_{i_{1}, \ldots, i_{k}}^{j} \in \mathbb{K}$ and $b_{i_{1}}^{j}, \ldots, b_{i_{k}}^{j} \in A$ then

$$
\begin{aligned}
& \partial_{d}^{\varepsilon_{0}, \ldots, \varepsilon_{d}}\left(a_{j}\right)=\sum_{i_{1}, \ldots, i_{k}} \sum_{j_{1}<j_{2}<\cdots<j_{d}} x_{i_{1}, \ldots, i_{k}}^{j} \varepsilon_{0}\left(b_{i_{1}}^{j}\right) \cdots \varepsilon_{0}\left(b_{i_{j_{1}-1}}^{j}\right) \\
& \quad \times \varepsilon_{1}\left(b_{i_{j_{1}+1}}^{j}\right) \cdots \varepsilon_{d-1}\left(b_{i_{j_{d}-1}}^{j}\right) \varepsilon_{d}\left(b_{i_{j_{d}+1}}^{j}\right) \cdots \varepsilon_{d}\left(b_{i_{k}}^{j}\right) \cdot b_{i_{j_{1}}}^{j} b_{i_{j_{2}}}^{j} \cdots b_{i_{j_{d}}}^{j}
\end{aligned}
$$

and

$$
\begin{aligned}
& \mu_{\varepsilon_{d}, \ldots, \varepsilon_{0}}^{d}\left(b_{d}, \ldots, b_{1}\right)=\sum_{a_{j} \in A} \sum_{l_{d}<\cdots<l_{1}} \sum_{\substack{b_{d}=b_{i_{l}}^{j}, \ldots, b_{1}=b_{1}=b_{i_{1}}^{j} \\
i_{1}, i_{1}}} x_{i_{1}, \ldots, i_{k}}^{j} \varepsilon_{d}\left(b_{i_{1}}^{j}\right) \cdots \varepsilon_{d}\left(b_{i_{l_{d}-1}}^{j}\right) \\
& \varepsilon_{d-1}\left(b_{i_{l_{d}+1}}^{j}\right) \cdots \varepsilon_{0}\left(b_{i_{l_{1}+1}}^{j}\right) \cdots \varepsilon_{0}\left(b_{i_{k}}^{j}\right) \cdot a_{j}
\end{aligned}
$$

where we do a slight abuse and identify $a_{j}$ with its dual generator. 
To make things clearer, if $w_{d} b_{d} w_{d-1} b_{d-1} w_{d-2} \cdots w_{1} b_{1} w_{0}$ is a word in $\partial(a)$ then we have a corresponding contribution $\varepsilon_{d}\left(w_{d}\right) \cdots \varepsilon_{1}\left(w_{1}\right) \varepsilon_{0}\left(w_{0}\right) \cdot a$ in $\mu_{\varepsilon_{d}, \ldots, \varepsilon_{0}}^{d}\left(b_{d}, \ldots, b_{1}\right)$ (see Figures 1 and 2 ). When obvious we will drop the subscripts and write $\mu^{d}$ instead of $\mu_{\varepsilon_{d}, \ldots, \varepsilon_{0}}^{d}$.

The most useful special cases of equations (2.3) and (2.4) are when $d=1$ which gives

$$
d^{\varepsilon_{0}, \varepsilon_{1}}\left(a_{j}\right)=\sum_{i_{1}, \ldots, i_{k}} \sum_{l=1, \ldots, k} x_{i_{1}, \ldots, i_{k}}^{j} \varepsilon_{0}\left(b_{i_{1}}^{j}\right) \cdots \varepsilon_{0}\left(b_{i_{l}-1}^{j}\right) \varepsilon_{1}\left(b_{i_{l}+1}^{j}\right) \cdots \varepsilon_{1}\left(b_{i_{k}}^{j}\right) \cdot b_{i_{l}}^{j}
$$

$$
\mu_{\varepsilon_{1}, \varepsilon_{0}}^{1}(b)=\sum_{\substack{a_{j} \in A \\ i_{1}, \ldots, i_{k} \\ b=b_{i_{l}}^{j}}} x_{i_{1}, \ldots, i_{k}}^{j} \varepsilon_{1}\left(b_{i_{1}}^{j}\right) \cdots \varepsilon_{1}\left(b_{i_{l}-1}^{j}\right) \varepsilon_{0}\left(b_{i_{l}+1}^{j}\right) \cdots \varepsilon_{0}\left(b_{i_{k}}^{j}\right) \cdot a_{j} .
$$

The homological category of $\operatorname{Aug}(A)$ is $H(\operatorname{Aug}(A))$ whose objects are the same as those of $\operatorname{Aug}(A)$, the morphism spaces are $\operatorname{Hom}\left(\varepsilon_{0}, \varepsilon_{1}\right)=$ $H\left(C_{\varepsilon_{1}, \varepsilon_{0}}\right)$ and the composition of morphisms is given by $\left[b_{1}\right] \circ\left[b_{2}\right]=$ $(-1)^{\left|b_{2}\right|}\left[\mu_{\varepsilon_{2}, \varepsilon_{1}, \varepsilon_{0}}^{2}\left(b_{2}, b_{1}\right)\right]$ (note the case $d=2$ of equation (2.2) implies that this is well defined). The case $d=3$ of equation (2.4) implies that this composition is associative, hence (except for the existence of an identity morphism) $H(\operatorname{Aug}(A))$ is a genuine category.

2.3. Equivalence of non-unital $\mathcal{A}_{\infty}$-categories. By the stabilization process for the grading when going from the world of DGA to the one of $\mathcal{A}_{\infty}$-category, generators of degree 0 in the morphism spaces are elements $a$ of $A$ such that $\operatorname{gr}(a)=-1$. This implies that, by degree considerations, the augmentation category has no reason for being unital (in any of the sense of [17]). The notion of equivalence of categories in this context is not immediate. For convenience for the reader we will recall here the basic definitions of $\mathcal{A}_{\infty}$-functors and natural transformations from [17]. Then we will propose a definition for equivalence of $\mathcal{A}_{\infty}$-categories which do not necessarily have units (cohomological or strict). For objects $\varepsilon_{0}$ and $\varepsilon_{1}$ in an $\mathcal{A}_{\infty}$-category $\mathcal{A}$ we maintain the notation $C_{\varepsilon_{1}, \varepsilon_{0}}$ for the morphism space from $\varepsilon_{0}$ to $\varepsilon_{1}$. As we will consider several categories, we will sometimes denote this morphism space $C_{\varepsilon_{1}, \varepsilon_{0}}^{\mathcal{A}}$ in order to specify in which category the morphism space is considered.

Definition 2.2. An $\mathcal{A}_{\infty}$-functor $\mathcal{F}$ between two $\mathcal{A}_{\infty}$-categories $\left(\mathcal{A},\left\{\mu_{\mathcal{A}}^{d}\right\}\right)$ and $\left(\mathcal{B},\left\{\mu_{\mathcal{B}}^{d}\right\}\right)$ consists of the following:

- A map $F: O b(\mathcal{A}) \rightarrow O b(\mathcal{B})$, 
- For each $d \geq 1$ and $\left(\varepsilon_{0}, \varepsilon_{1}, \ldots, \varepsilon_{d}\right) \in O b(\mathcal{A})^{d+1}$, a map $F_{\varepsilon_{d}, \ldots, \varepsilon_{0}}^{d}$ : $C_{\varepsilon_{d}, \varepsilon_{d-1}} \otimes \cdots \otimes C_{\varepsilon_{1}, \varepsilon_{0}} \rightarrow C_{F\left(\varepsilon_{d}\right), F\left(\varepsilon_{0}\right)}[1-d]$ satisfying:

$$
\begin{aligned}
& \sum_{r=1}^{d} \sum_{s_{1}+\cdots+s_{r}=d} \mu_{\mathcal{B}}^{r}\left(F_{\varepsilon_{d}, \ldots, \varepsilon_{d-s_{r}}}^{s_{r}}\left(a_{d}, \ldots, a_{d-s_{r}+1}\right), \ldots, F_{\varepsilon_{s_{1}}, \ldots, \varepsilon_{0}}^{s_{1}}\left(a_{s_{1}}, \ldots, a_{1}\right)\right) \\
& \quad=\sum_{i=1}^{d} \sum_{j=0}^{d-i}(-1)^{\mathbf{s}_{j}} F_{\varepsilon_{d}, \ldots, \varepsilon_{j+i}, \varepsilon_{j}, \ldots, \varepsilon_{0}}^{d-i+1}\left(a_{d}, \ldots, \mu_{\mathcal{A}}^{i}\left(a_{j+i}, \ldots, a_{j+1}\right), \ldots, a_{1}\right)
\end{aligned}
$$

The terms $d=1,2$ of equation (2.7) imply that $F_{\varepsilon_{1}, \varepsilon_{0}}^{1}$ descends to a map $H\left(F^{1}\right): H\left(C_{\varepsilon_{1}, \varepsilon_{0}}\right) \rightarrow H\left(C_{F\left(\varepsilon_{1}\right), F\left(\varepsilon_{0}\right)}\right)$ which is a functor from $H(\mathcal{A})$ to $H(\mathcal{B})$. Again we drop the subscripts from the notation as they are most of the time obvious and write $F^{d}$ for $F_{\varepsilon_{d}, \ldots, \varepsilon_{0}}^{d}$.

We now recall the definition of a pre-natural transformation between $\mathcal{A}_{\infty}$ functors.

Definition 2.3. Let $\mathcal{F}$ and $\mathcal{G}$ be two $\mathcal{A}_{\infty}$-functors from $\mathcal{A}$ to $\mathcal{B}$. A prenatural transformation of degree $g$ from $\mathcal{F}$ to $\mathcal{G}$ is a family $T=\left\{T^{d}\right\}_{d \geq 0}$ where each $T^{d}$ consists of maps $T_{\varepsilon_{d}, \ldots, \varepsilon_{0}}^{d}: C_{\varepsilon_{d}, \varepsilon_{d-1}} \otimes \cdots \otimes C_{\varepsilon_{1}, \varepsilon_{0}} \rightarrow$ $C_{G\left(\varepsilon_{d}\right), F\left(\varepsilon_{0}\right)}[g-d]$.

The set of pre-natural transformations comes with a degree 1 differential defined by

$$
\begin{aligned}
\mu^{1}(T)^{d}= & \sum_{r=1}^{d+1} \sum_{i=1}^{r} \sum_{s_{1}+\cdots+s_{r}=d}(-1)^{\dagger} \mu_{\mathcal{B}}^{r}\left(G^{s_{r}}\left(a_{d}, \ldots, a_{d-s_{r}+1}\right), \ldots,\right. \\
& G^{s_{i+1}}\left(a_{s_{1}+\cdots+s_{i+1}}, \ldots, a_{s_{1}+\cdots+s_{i}+1}\right), \\
& T_{\varepsilon_{s_{1}+\cdots+s_{i}}, \ldots, \varepsilon_{s_{1}+\cdots+s_{i-1}}}^{s_{i}}\left(a_{s_{1}+\cdots+s_{i}}, \ldots, a_{s_{1}+\cdots+s_{i-1}+1}\right), \\
& \left.F^{s_{i-1}}\left(a_{s_{1}+\cdots+s_{i-1}}, \ldots, a_{s_{1}+\cdots+s_{i-2}+1}\right), \ldots, F^{s_{1}}\left(a_{s_{1}}, \ldots, a_{1}\right)\right) \\
& -\sum_{i=1}^{d} \sum_{j=0}^{d-i}(-1)^{\mathcal{H}_{j}+g-1} T_{\varepsilon_{d}, \cdots, \varepsilon_{j+i}, \varepsilon_{j}, \ldots, \varepsilon_{0}}^{d-i+1}\left(a_{d}, \ldots, a_{j+i+1},\right. \\
& \left.\mu_{\mathcal{A}}^{i}\left(a_{j+i}, \ldots, a_{j+1}\right), a_{j}, \ldots, a_{1}\right)
\end{aligned}
$$

where $\dagger=(g-1)\left(\left|a_{1}\right|+\cdots+\left|a_{s_{1}+\cdots+s_{i-1}}\right|-s_{1}-\cdots-s_{i-1}\right)$.

A natural transformation is a pre-natural transformation such that $\mu^{1}(T)=0$. For convenience of the reader we will explicitly detail the case $d=0,1$ and 2 of $\mu^{1}(T)^{d}=0$ as it will be useful later.

- $d=0$ :

$$
T^{0} \in C_{G\left(\varepsilon_{0}\right), F\left(\varepsilon_{0}\right)} \text { s.t. } \mu^{1}\left(T^{0}\right)=0,
$$


- $d=1$ : $\mu^{2}\left(G\left(a_{1}\right), T^{0}\right)+(-1)^{(g-1)\left(\left|a_{1}\right|-1\right)} \mu^{2}\left(T^{0}, F\left(a_{1}\right)\right)+\mu^{1}\left(T^{1}\left(a_{1}\right)\right)-T^{1}\left(\mu^{1}\left(a_{1}\right)\right)=0$,

- $d=2$ :

$$
\begin{aligned}
& \mu^{3}\left(G^{1}\left(a_{2}\right), G^{1}\left(a_{1}\right), T^{0}\right)+(-1)^{(g-1)\left(\left|a_{1}\right|-1\right)} \mu^{3}\left(G^{1}\left(a_{2}\right), T^{0}, F^{1}\left(a_{1}\right)\right) \\
& \quad+(-1)^{(g-1)\left(\left|a_{1}\right|+\left|a_{2}\right|\right)} \mu^{3}\left(T^{0}, F^{1}\left(a_{1}\right), F^{1}\left(a_{2}\right)\right) \\
& \quad+\mu^{2}\left(G^{2}\left(a_{2}, a_{1}\right), T^{0}\right)+(-1)^{(g-1)\left(\left|a_{1}\right|+\left|a_{2}\right|\right)} \mu^{2}\left(T^{0}, F^{2}\left(a_{2}, a_{1}\right)\right) \\
& \quad+\mu^{2}\left(G^{1}\left(a_{2}\right), T^{1}\left(a_{1}\right)\right)+(-1)^{(g-1)\left(\left|a_{1}\right|-1\right)} \mu^{2}\left(T^{1}\left(a_{2}\right), F^{1}\left(a_{1}\right)\right) \\
& \quad+\mu^{1}\left(T^{2}\left(a_{2}, a_{1}\right)\right)(-1)^{g} T^{2}\left(a_{2}, \mu^{1}\left(a_{1}\right)\right)+(-1)^{g+\left|a_{1}\right|-1} T^{2}\left(\mu^{1}\left(a_{2}\right), a_{1}\right) \\
& \quad+(-1)^{g} T^{1}\left(\mu^{2}\left(a_{2}, a_{1}\right)\right)=0 .
\end{aligned}
$$

Equation (2.9) implies that $T^{0}$ descends to a family of maps from $F\left(\varepsilon_{0}\right)$ to $G\left(\varepsilon_{0}\right)$ in the homological category $\left(\left[T_{\varepsilon_{0}}^{0}\right] \in H\left(C_{G\left(\varepsilon_{0}\right), F\left(\varepsilon_{0}\right)}\right)\right)$ which by $(2.10)$ satisfies $H\left(G^{1}\right)([a]) \circ\left[T_{\varepsilon_{0}}^{0}\right]=(-1)^{g \cdot|a|}\left[T_{\varepsilon_{0}}^{0}\right] \circ H\left(F^{1}\right)([a])$, i.e., $\left[T^{0}\right]$ is a natural transformation from $H\left(F^{1}\right)$ to $H\left(G^{1}\right)$.

Now assume that $T^{0}=0$. Then equation (2.10) implies that $T_{\varepsilon_{1}, \varepsilon_{0}}^{1}$ : $C_{\varepsilon_{1}, \varepsilon_{0}} \rightarrow C_{G\left(\varepsilon_{1}\right), F\left(\varepsilon_{0}\right)}[g-1]$ is a chain map inducing $\left[T_{\varepsilon_{1}, \varepsilon_{0}}^{1}\right]$ in homology.

Following $[\mathbf{1 7}]$ we denote by nu-fun $(\mathcal{F}, \mathcal{G})^{g}$ the set of pre-natural transformations from $\mathcal{F}$ to $\mathcal{G}$. It comes with a filtration where $F^{r}\left(\operatorname{nu}-\operatorname{fun}(\mathcal{F}, \mathcal{G})^{g}\right)$ are pre-natural transformations such that $T^{k}=0$ for $k \leq r$. It is obvious from equation (2.8) that $\mu^{1}$ preserves this filtration. The previous remarks about the $T^{0}=0$ case is part of the statement in $[\mathbf{1 7}$, Section (1f)] that the first page of the spectral sequence associated to this filtration is

$$
\begin{aligned}
E_{1}^{r, g}= & \prod_{\varepsilon_{0}, \ldots, \varepsilon_{r}} \operatorname{Hom}_{\mathbb{K}}^{g}\left(\operatorname{Hom}_{H(\mathcal{A})}\left(\varepsilon_{r-1}, \varepsilon_{r}\right) \otimes \cdots \otimes \operatorname{Hom}_{H(\mathcal{A})}\left(\varepsilon_{1}, \varepsilon_{0}\right),\right. \\
& \operatorname{Hom}_{H(\mathcal{B})}\left(F\left(\varepsilon_{0}\right), G\left(\varepsilon_{r}\right)\right) .
\end{aligned}
$$

2.3.1. Yoneda modules. Recall from [17] that given an object $\varepsilon$ of an $\mathcal{A}_{\infty}$-category $\mathcal{A}$ one can see the assignment $\varepsilon_{1} \mapsto C_{\varepsilon, \varepsilon_{1}}$ as being part of the definition of a right $\mathcal{A}_{\infty}$-module over $\mathcal{A}$, called the right Yoneda module of $\varepsilon$ and denoted by $\mathcal{Y}_{r}^{\varepsilon}$. The action $\mu_{\mathcal{Y}_{r}^{\varepsilon}}^{d}: \mathcal{Y}_{r}^{\varepsilon}\left(\varepsilon_{d-1}\right) \otimes C_{\varepsilon_{d-1}, \varepsilon_{d-2}} \otimes \cdots \otimes C_{\varepsilon_{1}, \varepsilon_{0}} \rightarrow$ $\mathcal{Y}_{r}^{\varepsilon}\left(\varepsilon_{0}\right)$ of $\mathcal{A}$ on $\mathcal{Y}_{r}^{\varepsilon}$ is given by the $\mathcal{A}_{\infty}$ operation $\mu^{d}$ (see $[\mathbf{1 7}$, Section (11)]). This assignment is part of an $\mathcal{A}_{\infty}$-functor, called the Yoneda embedding and denoted by $\mathcal{Y}_{r}$, from $\mathcal{A}$ to the $\mathcal{A}_{\infty}$-category of right modules over $\mathcal{A}$. This functor is cohomologically faithful, it however fails to be cohomologically full if the category $\mathcal{A}$ lacks a cohomological unit (one cannot construct the retraction of $[\mathbf{1 7}$, Section $(\mathbf{2 g})])$. 
Let $\varepsilon$ and $\varepsilon^{\prime}$ be two objects of an $\mathcal{A}_{\infty}$-category $\mathcal{A}$, we say that $\varepsilon$ is pseudoisomorphic to $\varepsilon^{\prime}$ if there exists a quasi-isomorphism of $\mathcal{A}_{\infty}$-modules $T: \mathcal{Y}_{r}^{\varepsilon} \rightarrow$ $\mathcal{Y}_{r}^{\varepsilon^{\prime}}$ (i.e., $T$ induces an isomorphism in homology, see Definition 2.4). We will consider two $\mathcal{A}_{\infty}$-categories $\mathcal{A}$ and $\mathcal{B}$ to be pseudo-equivalent if there exists a cohomologically full and faithful functor $\mathcal{F}: \mathcal{A} \rightarrow \mathcal{B}$ such that any object of $\mathcal{B}$ is pseudo-isomorphic to an object of the form $F(\varepsilon)$. In this situation, $\mathcal{F}$ will be referred to as a pseudo-equivalence of $\mathcal{A}_{\infty}$-categories. Of course, when both involved categories admit cohomological units the definition recovers the one from [17] since the Yoneda embedding is cohomologically full and the last part of the definition implies that $H(\mathcal{F})$ is essentially surjective.

2.3.2. Cohomological adjunctions. In order to prove that stable isomorphic DGAs have pseudo-equivalent augmentation categories, one needs to prove that the relation of pseudo-equivalence is an equivalence relation. In order to do so we will introduce the notion of cohomologically adjoint functors and cohomological adjunction.

It is worthwhile to unravel the definition of morphism of modules in the $\mathcal{A}_{\infty}$ sense. A morphism of Yoneda modules is given by a sequence $T^{d}$ : $\mathcal{Y}_{r}^{\varepsilon}\left(\varepsilon_{d-1}\right) \otimes C_{\varepsilon_{d-1}, \varepsilon_{d-2}} \otimes \cdots \otimes C_{\varepsilon_{1}, \varepsilon_{0}} \rightarrow \mathcal{Y}_{r}^{\varepsilon^{\prime}}\left(\varepsilon_{0}\right), d \geq 1$, i.e., a family of maps

$$
T^{d}: C_{\varepsilon, \varepsilon_{d-1}} \otimes C_{\varepsilon_{d-1}, \varepsilon_{d-2}} \otimes \cdots \otimes C_{\varepsilon_{1}, \varepsilon_{0}} \rightarrow C_{\varepsilon^{\prime}, \varepsilon_{0}},
$$

such that (see $[\mathbf{1 7}$, equation $(\mathbf{1 . 2 1})]$ ):

$$
\begin{aligned}
& \sum_{j=0}^{d-1}(-1)^{\dagger} \mu^{j+1}\left(T^{d-j}\left(a_{d}, a_{d-1}, \ldots, a_{j+1}\right), a_{j}, \ldots, a_{1}\right) \\
& \quad+\sum_{i=1}^{d} \sum_{j=0}^{d-i}(-1)^{\dagger} T^{d-i+1}\left(a_{d}, a_{d-1}, \ldots, \mu^{i}\left(a_{j+i}, \ldots, a_{j+1}\right), a_{j}, \ldots, a_{1}\right)=0
\end{aligned}
$$

For $d=1$, equation (2.12) implies that $T^{1}$ is a chain map, we recall now the definition of quasi-isomorphism.

Definition 2.4. A morphism $T$ between two Yoneda modules $\mathcal{Y}_{r}^{\varepsilon}$ and $\mathcal{Y}_{r}^{\varepsilon^{\prime}}$ is a quasi-isomorphism if for every object $\varepsilon_{0}$ the chain map $T_{\varepsilon_{0}}^{1}: C_{\varepsilon, \varepsilon_{0}} \rightarrow C_{\varepsilon^{\prime}, \varepsilon_{0}}$ induces an isomorphism in homology.

Let $\mathcal{F}$ be a pseudo-equivalence from $\mathcal{A}$ to $\mathcal{B}$ then for any object $\varepsilon$ of $\mathcal{B}$ there is an object of $\mathcal{A}$ that we denote by $G(\varepsilon)$ such that $\varepsilon$ is pseudo-isomorphic to $F(G(\varepsilon))$. Considering all together the pseudo-isomorphism leads to a family of maps

$$
T^{d}: C_{\varepsilon_{d}, \varepsilon_{d-1}} \otimes \cdots \otimes C_{\varepsilon_{1}, \varepsilon_{0}} \rightarrow C_{F\left(G\left(\varepsilon_{d}\right)\right), \varepsilon_{0}}
$$


It follows from [17, Theorem 2.9] adapted in our context that $G$ is part of a cohomologically full and faithful functor $\mathcal{G}: \mathcal{B} \rightarrow \mathcal{A}$. Hence the family of maps $T^{d}$ is a pre-natural transformation $T \in F^{1}($ nu-fun $(I d, \mathcal{F} \circ \mathcal{G}))$. Comparing equations (2.8) and (2.12) we get that this pre-natural transformation satisfies:

$$
\begin{aligned}
\mu^{1}(T)^{d}= & \sum_{r=3}^{d} \sum_{s_{1}+\cdots+s_{r}=d}(-1)^{\dagger} \mu_{\mathcal{B}}^{r}\left((\mathcal{F} \circ \mathcal{G})^{s_{r}}\left(a_{d}, \ldots, a_{d-s_{r}+1}\right), \ldots\right. \\
& (\mathcal{F} \circ \mathcal{G})^{s_{3}}\left(a_{s_{1}+s_{2}+s_{3}}, \ldots, a_{s_{1}+s_{2}+1}\right) \\
& \left.T^{s_{2}}\left(a_{s_{1}+s_{2}}, \ldots, a_{s_{1}}\right), a_{s_{1}-1}, \ldots, a_{0}\right)
\end{aligned}
$$

Note that since $\mathcal{F}$ is cohomologically full and faithful it admits an inverse $\left[F^{1}\right]^{-1}$ in homology, since we work over a field this map actually lifts to a chain map $U^{1}: C_{F\left(\varepsilon_{1}^{\prime}\right), F\left(\varepsilon_{0}^{\prime}\right)}^{\mathcal{B}} \rightarrow C_{\varepsilon_{1}^{\prime}, \varepsilon_{0}^{\prime}}^{\mathcal{A}}$. The composition $U_{G\left(\varepsilon_{1}\right), \varepsilon_{0}^{\prime}}^{1} \circ T_{\varepsilon_{1}, F\left(\varepsilon_{0}^{\prime}\right)}^{1}$ : $C_{\varepsilon_{1}, F\left(\varepsilon_{0}^{\prime}\right)}^{\mathcal{B}} \rightarrow C_{G\left(\varepsilon_{1}\right), \varepsilon_{0}^{\prime}}^{\mathcal{A}}$ is a quasi-isomorphism inducing an isomorphism $\Theta$ : $\operatorname{Hom}\left(F\left(\varepsilon_{0}^{\prime}\right), \varepsilon_{1}\right) \simeq \operatorname{Hom}\left(\varepsilon_{0}^{\prime}, G\left(\varepsilon_{1}\right)\right)$. Combining equations $(2.11)$ (with $T^{0}=$ 0 ) and $(2.13)$ for the couple $\left(F^{1}(a), b\right)$ where $a \in C_{\varepsilon_{1}^{\prime}, \varepsilon_{0}^{\prime}}^{\mathcal{A}}$ and $b \in C_{\varepsilon_{0}, F\left(\varepsilon_{0}^{\prime}\right)}^{\mathcal{B}}$ one gets

$$
\mu_{\mathcal{B}}^{2}\left(T^{1}(b), F^{1}(a)\right)+T^{1}\left(\mu_{\mathcal{A}}^{2}\left(b, F^{1}(a)\right)=\mu^{1}\left(T^{2}\right)\left(b, F^{1}(a)\right)\right.
$$

This implies that $a$ induces the following commutative diagram in homology:

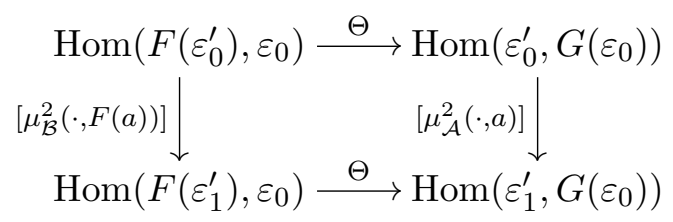

A similar diagram exists for left composition. All in all, the fact that $T$ induces an isomorphism of Yoneda modules and that $\mathcal{F}$ and $\mathcal{G}$ are cohomologically full and faithful implies that $T^{1}$ induces an adjunction between $H(F)$ and $H(G)$. This justifies the following definition.

Definition 2.5. Let $\mathcal{F}: \mathcal{A} \rightarrow \mathcal{B}$ and $\mathcal{G}: \mathcal{B} \rightarrow \mathcal{A}$ be two cohomologically full and faithful functors. A cohomological adjunction between $\mathcal{F}$ and $\mathcal{G}$ is a pre-natural transformation $T: I d \rightarrow \mathcal{F} \circ \mathcal{G}$ satisfying equation (2.13).

The previous discussion together with the adaptation of $[\mathbf{1 7}$, Theorem 2.9] in our context implies the following proposition. 
Proposition 2.6. An $\mathcal{A}_{\infty}$-functor $\mathcal{F}: \mathcal{A} \rightarrow \mathcal{B}$ is a pseudo-equivalence iff there exists a cohomologically full and faithful functor $\mathcal{G}: \mathcal{B} \rightarrow \mathcal{A}$ and a cohomological adjunction $T$ between $\mathcal{F}$ and $\mathcal{G}$.

We cannot emphasize enough that the fact that we work over a field is absolutely necessary for the last claim to be true, recall that in [17] one relies on the fact that we can split any complex as a sum of an acyclic complex and a complex with vanishing differential.

Note that the degree 1 pre-natural transformation $T: I d \rightarrow I d$ defined by $T^{1}=I d$ and $T^{d}=0$ for $d \neq 1$ satisfies $\mu^{1}(T)^{d}=\mu^{d}$ for $d$ even and 0 for $d$ odd and thus is a cohomological adjunction from $I d$ to $I d$. Under the geometric picture of Section 3.2, this should correspond to the interpolation between the two comparison maps given by the trivial cylinder which counts holomorphic curves with moving boundary conditions on (a small perturbation of) the trivial cylinder. In this situation, only the trivial curves contribute which gives the definition of $T^{1}$.

Note also that in the presence of $c$-unit, the evaluation $T^{1}(c)$ is an element of $C_{G\left(F\left(\varepsilon_{1}\right)\right), \varepsilon_{0}}$ which induces a natural isomorphism from $H(\mathcal{G} \circ \mathcal{F})$ to the identity as one expects from a quasi-equivalence in this context.

We are now able to prove the following proposition.

Proposition 2.7. The relation of pseudo-equivalence of $\mathcal{A}_{\infty}$-categories is an equivalence relation.

Proof. The identity functor is obviously a pseudo-equivalence as $T^{1}=I d$ induces an isomorphism from $\mathcal{Y}_{\varepsilon}^{r}$ to itself hence the relation is reflexive. It follows from Proposition 2.6 that it is symmetric. In order to prove transitivity let $\mathcal{F}_{0}: \mathcal{A} \rightarrow \mathcal{B}$ and $\mathcal{G}_{0}: \mathcal{B} \rightarrow \mathcal{A}$ be cohomologically adjoint pseudoequivalences. Similarly let $\mathcal{F}_{1}: \mathcal{B} \rightarrow \mathcal{C}$ be the pseudo-equivalence from $\mathcal{B}$ to $\mathcal{C}$ with cohomological adjoint $\mathcal{G}_{1}$. Denote by $T_{0}$ and $T_{1}$ the respective homological adjunctions.

$\mathcal{F}_{1}$ and $\mathcal{G}_{1}$ being cohomologically full and faithful, one gets that the map $H\left(F_{1}^{1}\right) \circ H\left(G_{1}^{1}\right)$ is an isomorphism. Again as we work over a field, this implies that there exists a chain map $S_{\varepsilon_{0}, \varepsilon_{1}}: C_{F_{1}\left(G_{1}\left(\varepsilon_{1}\right)\right), F_{1}\left(G_{1}\left(\varepsilon_{0}\right)\right)}^{\mathcal{C}} \rightarrow C_{\varepsilon_{1}, \varepsilon_{0}}^{\mathcal{C}}$ which induces $\left(H\left(F_{1}^{1}\right) \circ H\left(G_{1}^{1}\right)\right)^{-1}$ in homology.

This implies that the following composition,

$$
\begin{aligned}
& C_{\varepsilon_{1}, \varepsilon_{0}}^{\mathcal{C}} \stackrel{T_{0} \circ G_{1}}{\longrightarrow} C_{F_{0} \circ G_{0} \circ G_{1}\left(\varepsilon_{1}\right), G_{1}\left(\varepsilon_{0}\right)}^{\mathcal{B}} \stackrel{F_{1}}{\longrightarrow} C_{F_{1} \circ F_{0} \circ G_{0} \circ G_{1}\left(\varepsilon_{1}\right), F_{1} \circ G_{1}\left(\varepsilon_{0}\right)}^{\mathcal{C}} \\
& \quad \stackrel{S \circ T^{1}}{\longrightarrow} C_{F_{1} \circ F_{0} \circ G_{0} \circ G_{1}\left(\varepsilon_{1}\right), \varepsilon_{0}}^{\mathcal{C}}
\end{aligned}
$$

is a quasi-isomorphism. This is the first application necessary to define an adjunction from $\mathcal{F}_{1} \circ \mathcal{F}_{0}$ to $\mathcal{G}_{1} \circ \mathcal{G}_{0}$.

In order to define the higher order terms denote by $\mathcal{A}^{\prime}$ the image of $\mathcal{F}^{1} \circ \mathcal{G}^{1}$. The homological perturbation lemma of $[\mathbf{1 7}$, Section (1i)] allows us to 
extend $S$ to an $\mathcal{A}_{\infty}$-functor on $\mathcal{A}^{\prime}$. The homological adjunction is thus given by $\mu^{2}\left(\mathcal{L}_{S} T^{1}, \mathcal{L}_{F_{1}} \mathcal{R}_{G_{1}} T_{0}\right)$ where $\mu^{2}$ is the composition of natural transformations, $\mathcal{L}$ and $\mathcal{R}$ are respectively the left and right compositions of a prenatural transformation with a functor (see $[\mathbf{1 7}$, Section (1e)]). We are brief here as in the present paper only the homological result will be relevant and thus only the first map matters.

Remark 2.8. Note that at the most elementary level one gets that for an $\mathcal{A}_{\infty}$-category the set of isomorphism types of the groups $H\left(C_{\varepsilon_{1}, \varepsilon_{0}}\right)$ is invariant under quasi-equivalences.

Remark 2.9. Note that the map $S \circ T$ appearing in the proof of Proposition 2.7 induces an isomorphism from $\operatorname{Hom}\left(\varepsilon_{1}, F\left(\varepsilon_{0}^{\prime}\right)\right)$ to $\operatorname{Hom}\left(G\left(\varepsilon_{1}\right), \varepsilon_{0}^{\prime}\right)$ for any $\varepsilon_{0}^{\prime} \in \mathcal{A}$ and $\varepsilon_{1} \in \mathcal{B}$ whenever $T$ is a homological adjunction. This justifies the ambiguity between left and right adjunction.

\subsection{Invariance.}

2.4.1. Construction of functors on $\operatorname{Aug}(\boldsymbol{A})$. Let $\mathcal{A}$ and $\mathcal{B}$ be two semifree DGAs with generating sets $A$ and $B$ respectively and let $f: \mathcal{A} \rightarrow \mathcal{B}$ be a DGA map. We denote by $f_{n}$ the associated map between $\mathcal{A}_{n}$ and $\mathcal{B}_{n}$.

For an augmentation $\varepsilon$ set $F(\varepsilon)=\varepsilon \circ f$.

For a $n$-tuple of augmentations $E=\left(\varepsilon_{1}, \ldots, \varepsilon_{n}\right)$ one defines $f^{\varepsilon_{E}}: \mathcal{A}_{n} \rightarrow$ $\mathcal{B}_{n}$ by the $\mathbb{K}_{n}$-algebra morphism $\phi_{\varepsilon_{E}} \circ f_{n} \circ \phi_{F\left(\varepsilon_{E}\right)}^{-1}$ where $F\left(\varepsilon_{E}\right)$ is the n-uple $\left(F\left(\varepsilon_{1}\right), \ldots, F\left(\varepsilon_{n}\right)\right)$. This is a DGA-map from $\left(\mathcal{A}_{n}, \partial^{F\left(\varepsilon_{E}\right)}\right) \rightarrow\left(\mathcal{B}_{n}, \partial^{\varepsilon_{E}}\right)$. Its restriction to $C_{n}(A)$ decomposes as a sum $f_{1}^{\varepsilon_{E}} \oplus f_{2}^{\varepsilon_{E}} \oplus \cdots \oplus f_{j}^{\varepsilon_{E}} \oplus \cdots$ where $f_{j}^{\varepsilon_{E}}: C_{n}(A) \rightarrow C_{n}(B)^{\otimes j}$ are $\mathbb{K}_{n}$-bimodule homomorphisms. Note that there is no 0-order term in the decomposition as those coming from $F_{n}\left(\varepsilon_{E}\right)$ cancel with those coming from $\phi_{\varepsilon_{E} \circ f_{n}}^{-1}$. Dualizing each of the $f_{j}^{\varepsilon_{E}}$ and restricting them to $C_{\varepsilon_{d}, \varepsilon_{d-1}} \otimes \cdots \otimes C_{\varepsilon_{2}, \varepsilon_{1}}$ one gets maps $F_{\varepsilon_{E}}^{d}: C_{\varepsilon_{d}, \varepsilon_{d-1}} \otimes \cdots \otimes C_{\varepsilon_{2}, \varepsilon_{1}} \rightarrow$ $C_{F\left(\varepsilon_{d}\right), F\left(\varepsilon_{1}\right)}[1-d]$ (again note that here we use the absolute grading). As in Section 2.2.1 those only depend on the augmentations $\varepsilon_{1}, \ldots, \varepsilon_{d}$ and on $f$. We denote by $F^{d}$ the sets of maps $\left\{F_{\varepsilon_{d}, \ldots, \varepsilon_{1}}^{d}\right\}$ for all $d$-tuples of augmentations. From $f^{\varepsilon_{E}} \circ \partial^{F\left(\varepsilon_{E}\right)}=\partial^{\varepsilon_{E}} \circ f^{\varepsilon_{E}}$ one deduces the following

Proposition 2.10. The family $\mathcal{F}=\left\{F^{d}\right\}$ is an $A_{\infty}$-functor from $\operatorname{Aug}(B)$ to $\operatorname{Aug}(A)$.

The construction is functorial with respect to the composition as stated in the following:

Proposition 2.11. Let $f$ and $g$ be two DGA maps then the $\mathcal{A}_{\infty}$-functor associated to $f \circ g$ is $\mathcal{G} \circ \mathcal{F}$, where the composition of $\mathcal{A}_{\infty}$-functors is defined as in [17, Section (1b)]. 
Proof. Consider $\left.(f \circ g)^{\varepsilon_{E}}\right|_{C(A)}$. As $f$ is a DGA map one gets the decomposition

$$
\left.(f \circ g)^{\varepsilon_{E}}\right|_{C(A)}(a)=\sum \sum f_{s_{1}} \otimes \cdots \otimes f_{s_{r}}\left(g_{i}(a)\right) .
$$

Dualizing this equation and denoting $\mathcal{H}$ the functor associated to $f \circ g$ leads to the formula

$H^{d}\left(a_{d}, \ldots, a_{1}\right)=\sum_{r} \sum_{s_{1}+\cdots+s_{r}=d} G^{r}\left(F^{s_{r}}\left(a_{d}, \ldots, a_{d-s_{r}+1}\right), \ldots, F^{s_{1}}\left(a_{s_{1}}, \ldots, a_{1}\right)\right)$,

which is the composition formula of $\mathcal{A}_{\infty}$-functors.

2.4.2. Equivalence of augmentations. We are now ready to give the definition of equivalent augmentations.

Definition 2.12. Two augmentations $\varepsilon_{1}$ and $\varepsilon_{2}$ are equivalent if there is an $\mathcal{A}_{\infty}$-functor $\mathcal{F}$ such that $\varepsilon_{1}=F\left(\varepsilon_{2}\right)$ and a homological adjunction $T$ from $F$ to the identity.

Note that in [3] equivalence of augmentations has a different shape. Precisely it is said that two augmentations are equivalent if there exists a derivation $K$ such that $\varepsilon_{1}=\varepsilon_{2} \circ e^{K \partial+\partial K}$. This definition is of course problematic if our coefficient ring is not an algebra over a field of characteristic 0. Equivalent augmentations from $[\mathbf{3}]$ are equivalent in our sense using $f=e^{K \partial+\partial K}$ to construct the $\mathcal{A}_{\infty}$-functor. The natural transformation is given by dualizing homogenous component of $K$. It is not clear to us whether or not the converse is true, compare also Formula (2.12) with the formula appearing in [13, Lemma 3.13].

Definitions 2.4 and 2.9 imply the following

Theorem 2.13. Let $\varepsilon_{1}$ and $\varepsilon_{2}$ be two augmentations of $\mathcal{A}$. If $\varepsilon_{1}$ is equivalent to $\varepsilon_{2}$ then for any augmentation $\varepsilon$, we have $H\left(C_{\varepsilon_{1}, \varepsilon}\right) \simeq H\left(C_{\varepsilon_{2}, \varepsilon}\right)$ and $H\left(C_{\varepsilon, \varepsilon_{1}}\right) \simeq H\left(C_{\varepsilon, \varepsilon_{2}}\right)$.

2.4.3. Invariance under stable isomorphisms. Recall that a stabilization of a DGA is a new DGA with two more generators $b, c$ such that $\partial b=c$. Two DGAs are said to be stable isomorphic if they become isomorphic after a sequence of stabilizations. The aim of this paragraph is to prove the following:

Theorem 2.14. Let $\mathcal{A}$ and $\mathcal{A}^{\prime}$ be two stable isomorphic DGAs. Then their augmentation categories are pseudo-equivalent.

Proof. From Proposition 2.7, it is sufficient to prove the theorem first for an isomorphism and second for a stabilization. Let $f: \mathcal{A} \rightarrow \mathcal{A}$ be an isomorphism. Consider $\mathcal{F}$ the associated functor as in Proposition 2.10, and $\mathcal{G}$ the functor associated to $f^{-1}$. Then $\mathcal{F} \circ \mathcal{G}=I d$ and $\mathcal{G} \circ \mathcal{F}=I d$ imply that the categories $\operatorname{Aug}(\mathcal{A}, \partial)$ and $\operatorname{Aug}\left(\mathcal{A}, \partial^{\prime}\right)$ are pseudo-equivalent. 
Let $\mathcal{A}^{\prime}$ be a stabilization of $\mathcal{A}$. The natural inclusion $i: \mathcal{A} \rightarrow \mathcal{A}^{\prime}$ is a DGA morphism, inducing a functor $\mathcal{I}$. Note that here $I^{k}=0$ for $k \geq 2$. The map $j: \mathcal{A}^{\prime} \rightarrow \mathcal{A}$ which sends $b$ and $c$ to 0 is also a DGA morphism inducing $\mathcal{J}$. Obviously $\mathcal{I}$ and $\mathcal{J}$ are cohomologically full and faithful and $\mathcal{J} \circ \mathcal{I}$ is the identity. Note that as vector spaces the morphism spaces $C^{\varepsilon_{1}, \varepsilon_{2}}$ and $C^{\varepsilon_{1}, I \circ J\left(\varepsilon_{2}\right)}$ are canonically the same and that the augmented differential $\mu_{\varepsilon_{1}, \varepsilon_{2}}^{1}$ corresponds to the one for $I \circ J\left(\varepsilon_{i}\right)$. This implies that the pre-natural transformation defined under this identification by $T_{\varepsilon_{1}, \varepsilon_{2}}^{1}=I d$ and $T^{d}=0$ if $d \neq 1$ is a homological adjunction from $\mathcal{I}$ to $\mathcal{J}$ as noted in Section 2.3.

The set of equivalence classes of augmentations behave nicely with respect to stable isomorphisms of DGAs. Namely

Theorem 2.15. Let $\mathcal{A}(A)$ be a free $D G A$. Then we have:

(1) Let $f$ be an isomorphism of $\mathcal{A}$ then $\varepsilon_{1}$ is equivalent to $\varepsilon_{2}$ iff $\varepsilon_{1} \circ f$ is equivalent to $\varepsilon_{2} \circ f$.

(2) Let $i: \mathcal{A} \rightarrow \mathcal{A}^{\prime}$ be the inclusion of a DGA into one of his stabilizations. Then $\varepsilon_{1}$ is equivalent to $\varepsilon_{2}$ iff $\varepsilon_{1} \circ i$ is equivalent to $\varepsilon_{2} \circ i$ and two augmentations $\varepsilon_{1}^{\prime}, \varepsilon_{2}^{\prime}$ of $\mathcal{A}^{\prime}$ are equivalent iff their restrictions to $\mathcal{A}$ are.

Proof. The first part is obvious. It is sufficient to compose the natural adjunction with the functor induced by $f^{-1}$.

If the stabilization is not of degree 0 the result is obvious, thus we suppose that the stabilization has degree 0 . One needs to prove that if $\varepsilon^{\prime}$ is an augmentation of $\mathcal{A}^{\prime}$ then $\varepsilon_{0}^{\prime}$ defined by $\varepsilon_{0}^{\prime}(a)=\varepsilon^{\prime}(a)$ for all generators $a \in A$ and $\varepsilon_{0}^{\prime}(b)=\varepsilon_{0}^{\prime}(c)=0$ is equivalent to $\varepsilon^{\prime}$. To do so we proceed as in the proof of Theorem 2.14. We define the DGA morphism $f: \mathcal{A}^{\prime} \rightarrow \mathcal{A}^{\prime}$ by $f(a)=a$ for all $a \in A, f(b)=b-\varepsilon^{\prime}(b)$ and $f(c)=c$. Obviously $F\left(\varepsilon^{\prime}\right)=\varepsilon_{0}^{\prime}$. The prenatural transformation $T$ defined by $T^{1}=I d$ and $T^{d}=0$ induces the desired homological natural adjunction. The proof is complete as $\varepsilon_{0}^{\prime}=\left.\varepsilon^{\prime}\right|_{\mathcal{A}} \circ i$.

\section{Geometric interpretation}

3.1. Legendrian contact homology. In this section, we consider the jet space $\mathcal{J}^{1}(M):=T^{*} M \times \mathbb{R}$ of a $n$-dimensional manifold $M$ with the standard contact structure $\xi=\operatorname{ker}(d z-\theta)$ where $z$ parametrizes $\mathbb{R}$ and $\theta=\sum p d q$ is the standard Liouville form on $T^{*} M$ with $(q, p)$ the conjugate coordinates. A Legendrian submanifold $\Lambda$ of $\mathcal{J}^{1}(M)$ is a $n$-dimensional submanifold such that $T \Lambda \subset \xi$. We assume here that all Legendrian submanifolds are compact.

We denote by $\pi$ the canonical projection of $\mathcal{J}^{1}(M)$ to $T^{*} M$ and by $\Pi$ the canonical projection of $\mathcal{J}^{1}(M)$ to $M \times \mathbb{R}$. For a Legendrian submanifold $\Lambda, \pi(\Lambda)$ is called the Lagrangian projection and $\Pi(\Lambda)$ is called the front projection.

A Reeb chord $\gamma$ of $\Lambda$ is trajectory $\gamma:[0, T] \rightarrow \mathcal{J}^{1}(M)$ of $\frac{\partial}{\partial z}$ such that $T>0, \gamma(0)$ and $\gamma(T)$ belong to $\Lambda$. Those are in bijection with double 
points of the Lagrangian projection. A Reeb chord is non-degenerate if the corresponding double point of the Lagrangian projection is transverse and if all Reeb chords are non-degenerate $\Lambda$ is called chord generic. We denote by $\mathcal{R}(\Lambda)$ the set of Reeb chords of $\Lambda$ (if $\Lambda$ is chord generic this is a finite set since $\Lambda$ is compact).

If $\Lambda$ is chord-generic there is a grading map gr : $\mathcal{R}(\Lambda) \rightarrow \mathbb{Z}$ defined by the Conley-Zehnder index (see $[\mathbf{9}]$ ).

We denote by $C(\mathcal{R}(\Lambda))$ the graded $\mathbb{K}$-vector space generated by $\mathcal{R}(\Lambda)$ and $\mathcal{A}(\Lambda)$ the tensor algebra of $C(\mathcal{R}(\Lambda))$ as in Section 2 . In general, $\mathbb{K}$ will be $\mathbb{Z}_{2}$; if $\Lambda$ is relatively spin, one can also consider $\mathbb{Q}$. This algebra is called the Chekanov algebra of $\Lambda$, it is a DGA with differential $\partial$ defined counting some holomorphic curves in $\mathbb{R} \times \mathcal{J}^{1}(M)$ (see $\left.[\mathbf{1 0}, \mathbf{1 1}]\right)$.

Let $\widetilde{J}$ be an almost complex structure on $T^{*} M$ compatible with $-d \theta$. To $\widetilde{J}$ we associate an almost complex structure $J$ on $\mathbb{R} \times \mathcal{J}^{1}(M)$ by the following. The differential of the projection $\pi$ induces an isomorphism between $\left.\xi\right|_{(q, p, z)}$ and $T_{(q, p)}\left(T^{*} M\right)$; we set $\left.J\right|_{\xi}=\widetilde{J}$ under this identification. Finally, we set $J \frac{\partial}{\partial t}$ to be equal to $\frac{\partial}{\partial z}$. Such an almost complex structure will be referred to as a compatible almost complex structure.

We denote by $D_{k}$ the two-dimensional closed unit disk with the $(k+1)$ th roots of unity removed. For a complex structure $j$ on $D_{k}$ we choose holomorphic coordinates $[T, \infty] \times[0,1]$ near 1 and $[-\infty,-T] \times[0,1]$ near the other punctures. A map $u:\left(D_{k}, j\right) \rightarrow \mathbb{R} \times \mathcal{J}^{1}(M)$ is said to be holomorphic if $d u \circ j=J \circ d u$.

If $u$ is a holomorphic map then $\left.u\right|_{[T, \infty] \times[0,1]}$ decomposes as a map $(a, v, f)$ with $a:[T, \infty] \times[0,1] \rightarrow \mathbb{R}, v:[T, \infty] \times[0,1] \rightarrow T^{*} M$ and $f:[T, \infty] \times[0,1] \rightarrow$ $\mathbb{R}$. Suppose $u\left(\partial D_{k}\right) \subset \mathbb{R} \times \Lambda$ then for a Reeb chord $\gamma$ we say that $u$ has a positive asymptotic $\gamma$ at 1 if $v(z) \rightarrow \pi(\gamma)$ and $a(z) \rightarrow \infty$ when $z \rightarrow 1$. Similarly, for a root of unity $z_{0}$ we say that $u$ has a negative asymptotic $\gamma$ at $z_{0}$ if $v(z) \rightarrow \pi(\gamma)$ and $a(z) \rightarrow-\infty$ when $z \rightarrow z_{0}$.

For Reeb chords $\gamma^{+}, \gamma_{1}, \ldots, \gamma_{k}$ we denote by $\mathcal{M}\left(\gamma^{+}, \gamma_{1}, \ldots, \gamma_{k}\right)$ the moduli space of holomorphic maps from $\left(D_{k}, j\right)$ to $\mathbb{R} \times \mathcal{J}^{1}(M)$ with boundary on $\mathbb{R} \times \Lambda$, positive asymptotics $\gamma^{+}$and negative asymptotics $\gamma_{1}, \ldots, \gamma_{k}$ for all $j$ modulo biholomorphism $\left(D_{k}, j\right) \simeq\left(D_{k}, j^{\prime}\right)$ and translation in the $\mathbb{R}$ direction in $\mathbb{R} \times \mathcal{J}^{1}(M)$.

The differential $\partial$ on $\mathcal{A}(\Lambda)$ is defined by

$$
\partial \gamma^{+}=\sum_{\substack{\gamma_{1}, \ldots, \gamma_{k} \\ g r\left(\gamma_{1} \ldots \gamma_{k}\right)=g r\left(\gamma^{+}\right)-1}} \# \mathcal{M}\left(\gamma^{+}, \gamma_{1}, \ldots, \gamma_{k}\right) \cdot \gamma_{1} \ldots \gamma_{k}
$$

on generators and is extended to $\mathcal{A}(\Lambda)$ by linearity and the Leibniz rule.

The homology of the DGA $(\mathcal{A}(\Lambda), \partial)$ is called the Legendrian contact homology of $\Lambda$ and is denoted by $\operatorname{LCH}(\Lambda)$. 
As the Chekanov algebra (see $[\mathbf{5}, \mathbf{9}]$ ) of a Legendrian submanifold $\Lambda$ is a semi-free DGA, the previous construction applies in this case to give the augmentation category for the Legendrian submanifold $\Lambda$. We denote this category by $\operatorname{Aug}(\Lambda)$. In the next section, we will show that this augmentation category can be extracted from the geometrical data of the differential of the Chekanov algebra of the $n$-copy Legendrian link.

One can choose more sophisticated coefficient rings to define Legendrian contact homology, for instance one can keep track of the homology class of the curves defining the differential using the group ring $\mathbb{K}\left[H_{1}(\Lambda)\right]$. Even though this is not a field, one can still carry out the previous construction considering all the chain complexes, tensor products and dual spaces over $\mathbb{K}$ (the coefficients from $H_{1}(\Lambda)$ inducing a decomposition of the vector spaces).

\subsection{The augmentation category of Legendrian submanifolds.}

3.2.1. The augmentation category $\operatorname{Aug}(\boldsymbol{\Lambda})$. From Section 2, we deduce that there is an $\mathcal{A}_{\infty}$-category associated to $\mathcal{A}(\Lambda)$ we denote this category by $\operatorname{Aug}(\Lambda)$. Note that from Theorem 3.2 the curve contributing to the products $\mu^{n}$ are the curves shown in Figure 2 .

The homologies of the complexes $\left(C(\mathcal{R}(\Lambda)), d^{\varepsilon_{0}, \varepsilon_{1}}\right)$ and $\left(C(\mathcal{R}(L)), \mu_{\varepsilon_{1}, \varepsilon_{0}}^{1}\right)$ are called the bilinearized Legendrian contact homology and cohomology groups, and are denoted by $\mathrm{LCH}^{\varepsilon_{0}, \varepsilon_{1}}(L)$ and $\mathrm{LCH}_{\varepsilon_{1}, \varepsilon_{0}}(L)$.

Recall that Legendrian isotopies induce stable tame isomorphisms (and thus stable isomorphisms) of the Chekanov algebra (see $[\mathbf{5 , 9 , 1 1}]$ ). Thus Theorem 2.14 implies Theorems 1.2 and 1.1. Theorem 1.3 is itself a consequence of Theorem 2.13 which also implies Theorem 1.4.

We will now describe how the bilinearized differential appears naturally when considering the Chekanov algebra of the two-copy Legendrian link associated to $\Lambda$.

3.2.2. The $\boldsymbol{n}$-copy Legendrian link. Let $\Lambda$ be a Legendrian submanifold of $\mathcal{J}^{1}(M)$. Choose small Morse functions $f_{i}, i=2, \ldots, n$ on $\Lambda$ such that, for each $i \neq j$, the critical points of $f_{i}$ and $f_{j}$ are disjoint and $f_{i}-f_{j}$ is a Morse function. We also set $f_{1}=0$ in order to simplify notations. We denote by $\bar{\Lambda}_{n}$ the Legendrian link $\Lambda_{1} \sqcup \Lambda_{2} \sqcup \cdots \sqcup \Lambda_{n}$ where $\Lambda_{i}$ is a perturbation of $\Lambda+i \varepsilon \frac{\partial}{\partial z}$ by the 1-jet of the function $\varepsilon f_{i}$. The front and Lagrangian projections of $\bar{\Lambda}_{2}$ are illustrated by Figures 3 and 4 for the Legendrian unknot $\Lambda$.

3.2.3. Reeb chords of $\boldsymbol{\Lambda}_{\boldsymbol{n}}$. Let $\mathcal{R}(\Lambda)$ be the set of Reeb chords of $\Lambda$. Reeb chords of $\bar{\Lambda}_{n}$ are of three different types:

(1) Reeb chords of $\Lambda_{i}$ for each $i \in\{1, \ldots, n\}$ called pure chords.

(2) Critical points of $f_{i}-f_{j}$ for each $i \neq j$ called continuum chords.

(3) Long (i.e., not continuum) Reeb chords from $\Lambda_{i}$ to $\Lambda_{j}$ for each $i \neq j$ called mixed chords. 


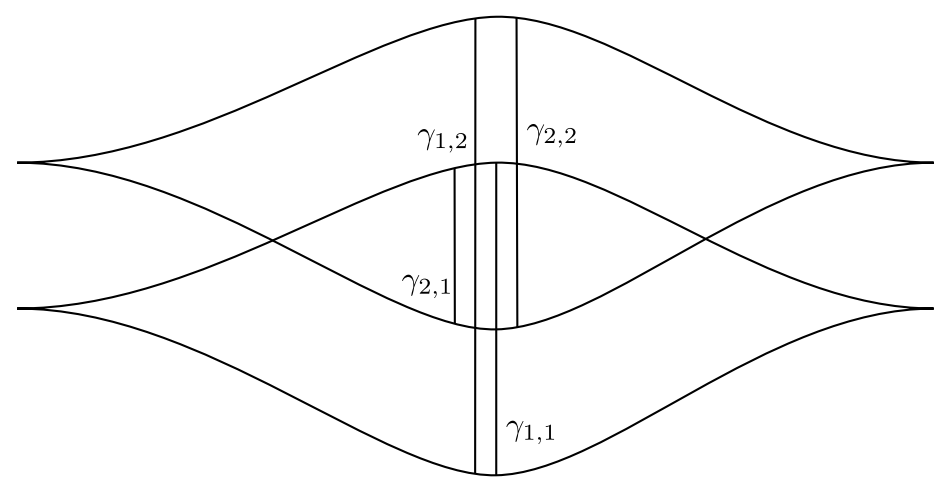

Figure 3. Front projection of the two-copy Legendrian unknot before perturbation.

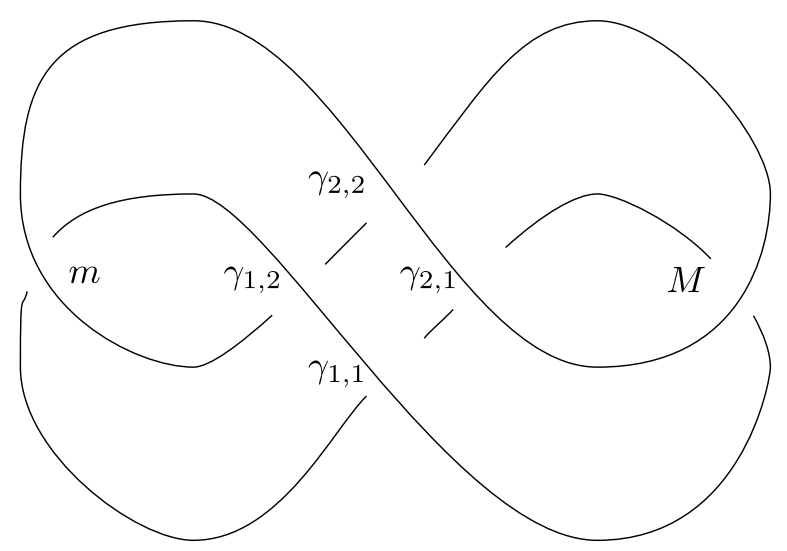

Figure 4. Lagrangian projection of the two-copy Legendrian unknot after perturbation.

For a fixed $i$, the set of chords of type 1 of $\Lambda_{i}$ are in bijection with $\mathcal{R}(\Lambda)$; for $\gamma \in \mathcal{R}(\Lambda)$ we denote $\gamma_{i, i}$ the corresponding chord of $\Lambda_{i}$. Similarly, for each $i \neq j$, chords of type 3 are in bijection with $\mathcal{R}(\Lambda)$ and for $\gamma \in \mathcal{R}(\Lambda)$ we denote by $\gamma_{i, j}$ the corresponding chord from $\Lambda_{i}$ to $\Lambda_{j}$.

3.2.4. The differential. Let $I_{c}$ be the two-sided ideal of $\mathcal{A}\left(\bar{\Lambda}_{n}\right)$ generated by continuum chords, i.e., Reeb chords coming from critical points of the functions $f_{i}-f_{j}$. Then we have:

Proposition 3.1. For sufficiently small $\varepsilon>0, \partial\left(I_{c}\right) \subset I_{c}$.

Proof. Let $\ell$ be the minimal length of all Reeb chords of type 1 and 3 and $F$ be the maximum of all maxima of the functions $f_{i}-f_{j}$ so that the length of any chord of type 2 is smaller than $\varepsilon(n-1+F)$. Set $\varepsilon<\frac{\ell}{n-1+F}$. As $\partial$ decreases the length of chords, we get that for any chord of type 2 its 
boundary is in the algebra generated by chords of type 2 . The Leibniz rule thus implies that $\partial\left(I_{c}\right) \subset I_{c}$.

We denote by $\mathcal{A}_{n}(\Lambda)$ the quotient $\mathcal{A}\left(\bar{\Lambda}_{n}\right) / I_{c}$. Then the differential on $\mathcal{A}\left(\bar{\Lambda}_{n}\right)$ descends to a differential $\partial_{n}$ on $\mathcal{A}_{n}(\Lambda)$. Note that $\mathcal{A}_{n}(\Lambda)$ is isomorphic to the tensor algebra over $\mathbb{K}$ of $C_{n}(\Lambda)$ where $C_{n}(\Lambda)$ is the free $\mathbb{K}$-vector space generated by Reeb chords of $\bar{\Lambda}_{n}$ of type 1 and 3 .

We extend the vector space structure of $C_{n}(\Lambda)$ to a $\mathbb{K}_{n}$-module structure by setting $e_{i} \cdot \gamma_{j, k} \cdot e_{l}=\delta_{i j} \cdot \delta_{k l} \cdot \gamma_{j, k}$.

3.2.5. Structure of $\boldsymbol{\partial}_{\boldsymbol{n}}$. Consider now the subalgebra of $\mathcal{A}_{n}(\Lambda)$ linearly generated by $\gamma_{i_{1}, j_{1}} \otimes \cdots \otimes \gamma_{i_{k}, j_{k}}$ where $\left(I=\left(i_{1}, \ldots, i_{k}\right), J=\left(j_{1}, \ldots, j_{k}\right)\right)$ is a composable pair of multi-indices. As a $\mathbb{K}_{n}$-algebra it is isomorphic to the algebra $\mathcal{A}_{n}(A)$ of Section 2.1 with $A=\mathcal{R}(\Lambda)$. As the differential $\partial$ counts connected holomorphic curves it is obvious that $\partial_{n}\left(\mathcal{A}_{n}(\mathcal{R}(\Lambda))\right) \subset \mathcal{A}_{n}(\mathcal{R}(\Lambda))$.

Theorem 3.2. For sufficiently small $\varepsilon>0,\left.\partial_{n}\right|_{\mathcal{A}_{n}(\mathcal{R}(\Lambda))}$ is the differential on the $n$-copy algebra of $(\mathcal{A}(\Lambda), \partial)$ as in Section 2.1 .

Proof. Consider $\bar{\Lambda}_{n}^{0}$ the $n$-copy Legendrian link consisting of $n$-linked copies of $\Lambda$ translated in the $z$-direction, without perturbing them by Morse functions. Reeb chords of this link are not all non-degenerate as any point of $\Lambda_{i}$ belongs to a chord running from $\Lambda_{i}$ to $\Lambda_{j}$ for all $j \neq i$. However, if chords of $\Lambda$ are non-degenerate then chords of $\bar{\Lambda}_{n}^{0}$ are Morse-Bott. Similarly to [2], one can consider the Morse-Bott approach to Legendrian contact homology using a Morse-Bott generic compatible almost complex structure $J$ on $\mathbb{R} \times M$.

For sufficiently small $\varepsilon>0, J$ will be a generic almost complex structure in the sense of $[\mathbf{1 1}]$ for the link $\bar{\Lambda}_{n}^{t}$ obtained by perturbing $\bar{\Lambda}_{n}^{0}$ using the Morse function $t \cdot f_{i}$, for all $t \in(0, \epsilon)$. All chords of $\bar{\Lambda}_{n}^{t}$ are described as before and are therefore in natural bijection for all $t \in(0, \epsilon)$. When $t=0$, all non-continuum chords are in bijection with non-continuum chords of $\bar{\Lambda}_{n}^{t}$ for $t>0$ and are non-degenerate. Thus, as they are all in natural bijection, we drop the index $t$ and denote them by the same letter.

Let $\mathcal{M}_{t}\left(\gamma^{+}, \gamma_{1}, \ldots, \gamma_{k}\right)$ be the moduli space of holomorphic maps on $D_{k}$ with boundary on $\mathbb{R} \times \bar{\Lambda}_{n}^{t}$. We label the connected components of $\partial\left(D_{k}\right)$ by $C_{0}, \ldots, C_{k+1}$ starting from the arc connecting 1 to $e^{2 \pi i /(k+1)}$. For a multiindex $I=\left(i_{0}, i_{1}, \ldots, i_{k}\right)$ we denote by $\mathcal{M}_{t}^{I}\left(\gamma^{+}, \gamma_{1}, \ldots, \gamma_{k}\right)$ the moduli space consisting of holomorphic maps such that the connected component $C_{j}$ of $\partial\left(D_{k}\right)$ is mapped to $\mathbb{R} \times \Lambda_{i_{j}}^{t}$, for $j=0, \ldots, k$.

If $\varepsilon>0$ is small enough, if $\gamma^{+}, \gamma_{1}, \ldots, \gamma_{k}$ are non-continuum chords, then the moduli spaces $\mathcal{M}_{t}^{I}\left(\gamma^{+}, \gamma_{1}, \ldots, \gamma_{k}\right)$ are diffeomorphic for all $t \in[0, \varepsilon)$, since all non-continuum chords are non-degenerate. 
Recall that elements of $\mathcal{M}^{I}\left(\gamma^{+}, \gamma_{1}, \ldots, \gamma_{j}\right)$ are in bijection with holomorphic curves in $T^{*} M$ with boundary on the Lagrangian projection of $\Lambda_{i}$ with punctures to the double points corresponding to the Reeb chords (see $[\mathbf{1 4}]$ ). As the Lagrangian projection of $\Lambda+i \varepsilon \frac{\partial}{\partial z}$ is equal to the Lagrangian projection of $\Lambda$, the moduli space of holomorphic curves $\mathcal{M}^{I}\left(\gamma^{+}, \gamma_{1}, \ldots, \gamma_{k}\right)$ and $\mathcal{M}^{J}\left(\gamma^{+}, \gamma_{1}, \ldots, \gamma_{k}\right)$ are in bijective correspondence for any multi-indices $I$ and $J$ of length $k+1$.

For a $n$-tuple of augmentations $E=\left(\varepsilon_{1}, \ldots, \varepsilon_{n}\right)$ of $\mathcal{A}(\Lambda)$ over $\mathbb{K}$ one gets an augmentation $\varepsilon_{E}$ of $\mathcal{A}\left(\bar{\Lambda}_{n}\right)$ over $\mathbb{K}_{n}$ setting $\varepsilon_{E}\left(\gamma_{i, i}\right)=\varepsilon_{i}(\gamma) \cdot e_{i}$ for any $\gamma \in \mathcal{R}(\Lambda)$ and sending every mixed chord to 0 similarly as done in Section 2.1.3. The fact that this is an augmentation follows from Theorem 3.2, Proposition 3.1 and applying Proposition 2.1 to the quotient algebra.

For $n=2$, the augmented complex decomposes as $C^{1,1} \oplus C^{2,2} \oplus C^{1,2} \oplus$ $C^{2,1} \oplus C^{1,2}(f)$ and from the fact that only the pure chords are augmented we get that $C^{i, i}$ are subcomplexes, the corresponding differential is the standard augmented differential. $C^{2,1}$ is also a subcomplex, it follows from Theorem 3.2 that its differential corresponds to the bilinearized differential $d^{\varepsilon_{1}, \varepsilon_{2}}$. Finally $C^{1,2} \oplus C^{1,2}(f)$ is a subcomplex with differential

$$
\left(\begin{array}{cc}
d^{\varepsilon_{1}, \varepsilon_{2}} & 0 \\
\rho & d^{f}
\end{array}\right) .
$$

The differential $d^{\varepsilon_{1}, \varepsilon_{2}}$ is the bilinearized differential and the complex $\left(C(f), d^{f}\right)$ is the Morse complex of $f_{2}$. This can be seen by a standard Morse-Bott argument and follows for example from [12, Theorem 3.6]. Finally, $\rho$ is a chain map and thus the complex $C^{1,2} \oplus C^{1,2}(f)$ is the cone over $\rho$, we denote it $C(\rho)$.

3.3. Duality exact sequence. A Legendrian submanifold $\Lambda$ of $\mathcal{J}^{1}(M)$ is horizontally displaceable if there exists a Hamiltonian diffeomorphism $\phi$ of $T^{*} M$ such that $\phi(\pi(\Lambda)) \cap \pi(\Lambda)=\emptyset$. Note that if $M$ is non-compact any compact Legendrian submanifold is horizontally displaceable.

In [12], it is proved that linearized Legendrian contact homology and cohomology of horizontally displaceable Legendrian submanifolds are parts of an exact sequence (which generalizes the duality of [16]):

$$
\cdots \rightarrow H_{k+1}(\Lambda) \rightarrow \mathrm{LCH}_{\varepsilon}^{n-k-1}(\Lambda) \rightarrow \mathrm{LCH}_{k}^{\varepsilon}(\Lambda) \rightarrow H_{k}(\Lambda) \rightarrow \cdots
$$

called the duality exact sequence.

The same considerations allow us to prove Theorem 1.5.

Proof of Theorem 1.5. This is almost a direct application of the arguments in [12] applied to the 4-component link $2 \bar{\Lambda}_{2}$ which consists of two copies $\bar{\Lambda}_{2}$ and $\widetilde{\Lambda}_{2}$ of $\bar{\Lambda}_{2}$ far apart in the $z$-direction (one perturbs the second copy using Morse functions in the same manner as in the previous section). 
First note that the discussion at the end of the previous section implies that there is an exact sequence induced by $\rho$ :

$$
\cdots \rightarrow H_{k+1}(\Lambda) \stackrel{i_{*}}{\rightarrow} H(C(\rho)) \stackrel{\pi_{*}}{\longrightarrow} \mathrm{LCH}_{k}^{\varepsilon_{1}, \varepsilon_{2}}(\Lambda) \stackrel{\rho}{\rightarrow} \cdots
$$

It remains to identify $H(C(\rho))$ with the bilinearized Legendrian cohomology. In order to do so, one must investigate how the exact sequence is built in [12]. One closely follows the notation from there. The Reeb chords of $2 \bar{\Lambda}_{2}$ are of four types:

(1) Chords going from $\bar{\Lambda}_{2}$ to itself denoted by $q^{0}$ and their corresponding chords on $\widetilde{\Lambda}_{2}$ denoted by $\widetilde{q^{0}}$.

(2) Chords going from $\bar{\Lambda}_{2}$ to $\widetilde{\Lambda_{2}}$ denoted by $q^{1}$ whose positive and negative ends are nearby the positive and negative ends of the corresponding chords on $\bar{\Lambda}_{2}$.

(3) Chords going from $\bar{\Lambda}_{2}$ to $\widetilde{\Lambda_{2}}$ denoted by $p^{1}$ whose positive ends are nearby the negative ends of the corresponding chords on $\widetilde{\Lambda_{2}}$ and negative ends are nearby the positive ends of the corresponding chords on $\bar{\Lambda}_{2}$.

(4) Critical points of the Morse functions on $\Lambda$ used to perturb $\widetilde{\Lambda}_{2}$.

The corresponding vector space complexes are denoted $Q^{0}, Q^{1}, P^{1}$ and $C^{1}$. Since $\bar{\Lambda}_{2}$ is itself a two-copy Legendrian link each of those vector spaces decomposes further. We label each of the summand by the positive and negative ends of the chords. For instance, $Q^{1}=Q_{11}^{1} \oplus Q_{22}^{1} \oplus Q_{12}^{1} \oplus Q_{21}^{1} \oplus Q_{f_{1}}^{1}$ where the differential splits as in the previous section. The vector space $Q^{1} \oplus$ $C^{1}$ is a subcomplex for the linearized differential given by the augmentation $\varepsilon_{12}$ of $2 \bar{\Lambda}_{2}$ given by $\varepsilon_{1}$ and $\varepsilon_{2}$. This subcomplex is shown to have the structure of a mapping cone of a map $\eta:\left(Q^{1}, d^{\varepsilon_{12}}\right) \rightarrow\left(C^{f}, d^{f}\right)$. With respect to the decomposition of $Q^{1}$ this map turns out to be $\eta=\rho_{11} \oplus \rho_{22} \oplus 0 \oplus 0 \oplus 0$; in other terms the vector space $Q_{12}^{1} \oplus Q_{21}^{1} \oplus Q_{f_{1}}^{1}$ is a subcomplex.

The long exact sequence of equation (3.1) is the one given by this mapping cone, this implies that the exact sequence for the $\operatorname{link} \bar{\Lambda}_{2}$ :

$$
\begin{aligned}
\cdots \rightarrow & H_{k+1}(\Lambda) \oplus H_{k+1}(\Lambda) \rightarrow \\
& \operatorname{LCH}_{\varepsilon_{1}}^{n-k-1}(\Lambda) \oplus \mathrm{LCH}_{\varepsilon_{2}}^{n-k-1}(\Lambda) \oplus \mathrm{LCH}_{\varepsilon_{2}, \varepsilon_{1}}^{n-k-1}(\Lambda) \oplus H^{n-k-1}\left(C\left(\rho^{*}\right)\right) \rightarrow \\
& \mathrm{LCH}_{k}^{\varepsilon_{1}}(\Lambda) \oplus \mathrm{LCH}_{k}^{\varepsilon_{2}}(\Lambda) \oplus \mathrm{LCH}_{k}^{\varepsilon_{1}, \varepsilon_{2}}(\Lambda) \oplus H_{k}(C(\rho)) \rightarrow \cdots
\end{aligned}
$$

splits as four exact sequences, the first two are the exact sequences for $\mathrm{LCH}^{\varepsilon_{1}}$ and $\mathrm{LCH}^{\varepsilon_{2}}$ and the other two lead to isomorphisms: $0 \rightarrow \mathrm{LCH}_{\varepsilon_{2}, \varepsilon_{1}}^{n-k-1}(\Lambda) \rightarrow$ $H_{k} C(\rho) \rightarrow 0$ and $0 \rightarrow H^{n-k-1}\left(C\left(\rho^{*}\right)\right) \rightarrow \mathrm{LCH}_{k}^{\varepsilon_{1}, \varepsilon_{2}}(\Lambda) \rightarrow 0$. This completes the proof.

A difference with the duality exact sequence lies in the fact that since $\varepsilon_{0}$ is a priori different from $\varepsilon_{1}$ the manifold class (as in [12]) is not well 
understood, this is due to the fact that one cannot describe the fundamental class (see Example 5.1).

\section{Perspectives}

4.1. Lagrangian fillings. In [8], Ekholm developed a Lagrangian intersection Floer theory for exact Lagrangian fillings related to the construction of the rational relative symplectic field theory of $[7]$. This construction is related to the wrapped Fukaya category of $[\mathbf{1}]$. The convex part of this theory is the Lagrangian intersection theory of the Lagrangian fillings in the classical sense.

We recall the definition of Lagrangian filling which is a special case of Lagrangian cobordism as in [4].

Definition 4.1. A Lagrangian filling of a Legendrian submanifold $\Lambda$ of a contact manifold $(Y, \xi)$ in $\mathbb{R} \times Y$ is a compact Lagrangian submanifold $L$ of $(-\infty, 0) \times Y$ such that

(i) $\partial L=\Lambda$,

(ii) $L \cup\left(\mathbb{R}_{+} \times \Lambda\right)$ is a Lagrangian submanifold of $\mathbb{R} \times Y$.

In $[\mathbf{8}, \mathbf{1 3}]$, it is shown that an exact Lagrangian filling with vanishing Maslov class of a Legendrian knot leads to an augmentation of its Chekanov algebra. We denote the augmentation induced by $L$ by $\varepsilon_{L}$. A consequence of the results of $[\mathbf{8}]$ is the following isomorphism:

$$
\operatorname{LCH}_{\varepsilon_{L}}^{n-k+2}(\Lambda) \simeq H_{k}(L) .
$$

The proof relies on the existence of an exact triangle involving $\mathrm{LCH}^{\varepsilon_{L}}(\Lambda)$, $H F(L)$ and the full Lagrangian Floer homology of $L$.

Similarly to the case of the wrapped Floer homology, in jet space the full Floer homology vanishes, so that $\mathrm{LCH}^{\varepsilon_{L}}(\Lambda)$ and $H F(L)$ are isomorphic. Standard arguments then show that $H F(L) \simeq H(L)$. In the context of bilinearized Legendrian contact homology, only the latter isomorphism fails to be true as two different Lagrangian fillings might not be Hamiltonian isotopic. Namely from [8, Theorem 4.9] we have:

Theorem 4.2. Let $L_{1}$ and $L_{2}$ be two exact Lagrangian fillings of $\Lambda$ with vanishing Maslov class inducing augmentations $\varepsilon_{L_{1}}$ and $\varepsilon_{L_{2}}$ of $\mathcal{A}(\Lambda)$. Then

$$
\operatorname{LCH}_{\varepsilon_{L_{1}}, \varepsilon_{L_{2}}}^{n-k+2}(\Lambda) \simeq H F^{k}\left(L_{1}, L_{2}\right)
$$

The proof is exactly the same as in $[\mathbf{8}$, Section 4.4$]$ but we make no use of the conjectural Lemma 4.10 from there as we do not need to compare holomorphic curves with Morse gradient trajectories here. 
4.2. Relation with generating families. This project is part of an attempt in producing a unified picture of the Legendrian invariants arising from holomorphic curves on one side and generating families on the other. Recall that a generating family for a Legendrian submanifold $\Lambda$ in jet space $\mathcal{J}^{1}(M)$ is a function $F: M \times \mathbb{R}^{m} \rightarrow \mathbb{R}$ such that $\Lambda=$ $\left\{\left(q_{0}, p_{0}, F\left(q_{0}, \eta_{0}\right)\right) \mid \frac{\partial F}{\partial \eta}\left(q_{0}, \eta_{0}\right)=0 p_{0}=\frac{\partial F}{\partial q}\left(q_{0}, \eta_{0}\right)\right\}$. To such a family one associates the difference function

$$
\begin{array}{cccc}
\widetilde{F}: & M \times \mathbb{R}^{m} \times \mathbb{R}^{m} & \rightarrow & \mathbb{R} \\
\left(q, \eta_{1}, \eta_{2}\right) & \mapsto & F\left(q, \eta_{2}\right)-F\left(q, \eta_{1}\right)
\end{array}
$$

and defines the generating family homology to be $G F(\Lambda, F)=H_{*}(F \geq$ $\epsilon, F=\epsilon)$. The set of those homology groups for all generating families form a Legendrian invariant bearing similarities with the linearized Legendrian contact homology. For instance, when $M=\mathbb{R}$, it has been shown in $[\mathbf{1 5}]$ that $\operatorname{LCH}^{\varepsilon}(\Lambda) \simeq G F\left(\Lambda, F^{\varepsilon}\right)$ where $F^{\varepsilon}$ is an equivalence class of generating family associated to $\varepsilon$ via graded ruling. It is a conjecture that this result holds in any dimension.

Bilinearized Legendrian contact homology appears naturally when trying to extend this conjectural isomorphism to a generalization of generating family homology to an invariant communicated to us by Petya Pushkar and defined using the function:

$$
\begin{array}{cccc}
\widetilde{F}: M \times \mathbb{R}^{m} \times \mathbb{R}^{m} & \rightarrow & \mathbb{R} \\
\left(q, \eta_{1}, \eta_{2}\right) & \mapsto & F_{1}\left(q, \eta_{2}\right)-F_{2}\left(q, \eta_{1}\right)
\end{array}
$$

and constructing $G F\left(\Lambda ; F_{1}, F_{2}\right)=H_{*}(\widetilde{F} \geq \epsilon, \widetilde{F}=\epsilon)$ where $F_{1}$ and $F_{2}$ are two generating families for $\Lambda$ (one assume that they have been stabilized to be defined on the same space). Conjecturally, those homologies correspond to the bilinearized Legendrian contact homology groups. More deeply, one can adapt a construction of Fukaya to construct an $\mathcal{A}_{\infty}$-category whose objects are generating families for $\Lambda$ and morphism spaces are the Morse complexes associated to $\widetilde{F}$. One expects the existence of an $\mathcal{A}_{\infty}$-functor from this category to $\operatorname{Aug}(\Lambda)$. It is not expected that this functor is a quasiequivalence, we however expect it to be cohomologically full and faithful and that it induces an equivalence of categories for the derived categories.

Note that the considerations in Section 4.1 also imply the existence of a cohomologically full and faithful functor from a Fukaya-type category constructed using a Lagrangian filling of $\Lambda$ to $\operatorname{Aug}(\Lambda)$ we similarly conjecture that this induces a quasi-equivalence of the derived categories.

\section{Examples}

In this section, we will show how bilinearized contact homology allows us to distinguish augmentations of some DGAs. 
5.1. Trefoil knot. We start with the example of the maximal ThurstonBennequin right-handed trefoil knot shown in Figure 5. It has five augmentations all of which lead to isomorphic linearized contact homologies. However, we will see that in the bilinearized contact homology table non-diagonal terms are not isomorphic to any diagonal term. As a consequence, all those five augmentations are pairwise non-homotopic. Moreover, all five augmentations arise from Lagrangian fillings of the trefoil knot. Thus, the computation implies that those Lagrangian fillings are pairwise non-symplectically equivalent (a result claimed by Ekholm, Honda and Kàlmàn in [13]).

The Chekanov algebra of $K$ has five generators. Two of degree 1: $a_{1}$ and $a_{2}$, and three of degree $0: b_{1}, b_{2}$ and $b_{3}$. The differential is given by:

$$
\begin{aligned}
& \partial a_{1}=1+b_{1}+b_{3}+b_{1} b_{2} b_{3}, \\
& \partial a_{2}=1+b_{1}+b_{3}+b_{3} b_{2} b_{1} .
\end{aligned}
$$

This DGA admits five augmentations listed in Table 1.

An easy computation shows that $\mathrm{LCH}^{\varepsilon_{i}, \varepsilon_{j}}(K)=\mathbb{Z}_{2}[0]$ as soon as $i \neq j$ and that $\mathrm{LCH}^{\varepsilon_{i}, \varepsilon_{i}}(K)=\mathbb{Z}_{2}[1] \oplus \mathbb{Z}_{2}^{2}[0]$. These five augmentations are thus all pairwise non-equivalent. As it is shown in [13], those five augmentations arise in this context from five different Lagrangian fillings of $K$ (one gets them

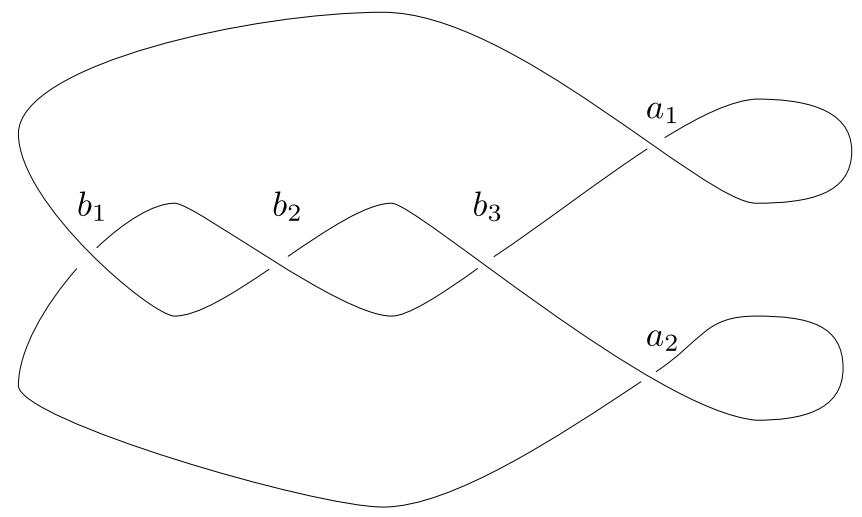

Figure 5. Right-handed maximal $t b$ trefoil knot.

Table 1. Augmentations of the right-handed maximal $t b$ trefoil knot

\begin{tabular}{cccc}
\hline & $b_{1}$ & $b_{2}$ & $b_{3}$ \\
\hline$\varepsilon_{1}$ & 1 & 1 & 1 \\
$\varepsilon_{2}$ & 1 & 0 & 0 \\
$\varepsilon_{3}$ & 1 & 1 & 0 \\
$\varepsilon_{4}$ & 0 & 0 & 1 \\
$\varepsilon_{5}$ & 0 & 1 & 1 \\
\hline
\end{tabular}


by resolving the crossing $b_{i}$ in various orders). It follows from our computation and Theorem 4.2 that those Lagrangian fillings are non-Hamiltonian isotopic. Note that it follows from [13, Lemma 3.13] that isotopic exact Lagrangian fillings lead to equivalent augmentations (the map $T^{d}$ is defined counting degree -1 holomorphic curves along a generic isotopy $\Sigma_{t}$ ).

We compute also the maps of the exact sequence of Section 3.3 in order to show that there is no fundamental class in the context of bilinearized Legendrian contact homology. To get the explicit maps one needs to consider the differential of the two-copy trefoil knot of Figure 6 (here the Morse function on $S^{1}$ has two minima $m_{1}$ and $m_{2}$ and two maxima $M_{1}$ and $M_{2}$ ). Specifically one needs to understand the map $\rho: C^{21} \rightarrow C^{f}$ of Section 3.2.5 arising while computing the differential $\partial$ on generators of type $\gamma_{12}$. From Figure 6 we get:

$$
\begin{aligned}
& \partial\left(a_{12}^{1}\right)=M_{1}+m_{1} a_{11}^{1}+(\cdots), \\
& \partial\left(a_{12}^{2}\right)=M_{2}+a_{22}^{2} m_{2}+(\cdots), \\
& \partial\left(b_{12}^{1}\right)=m_{1} b_{11}^{1}+b_{22}^{1} m_{2}, \\
& \partial\left(b_{12}^{2}\right)=m_{1} b_{11}^{2}+b_{22}^{2} m_{2}, \\
& \partial\left(b_{12}^{3}\right)=m_{1} b_{11}^{3}+b_{22}^{3} m_{2} .
\end{aligned}
$$

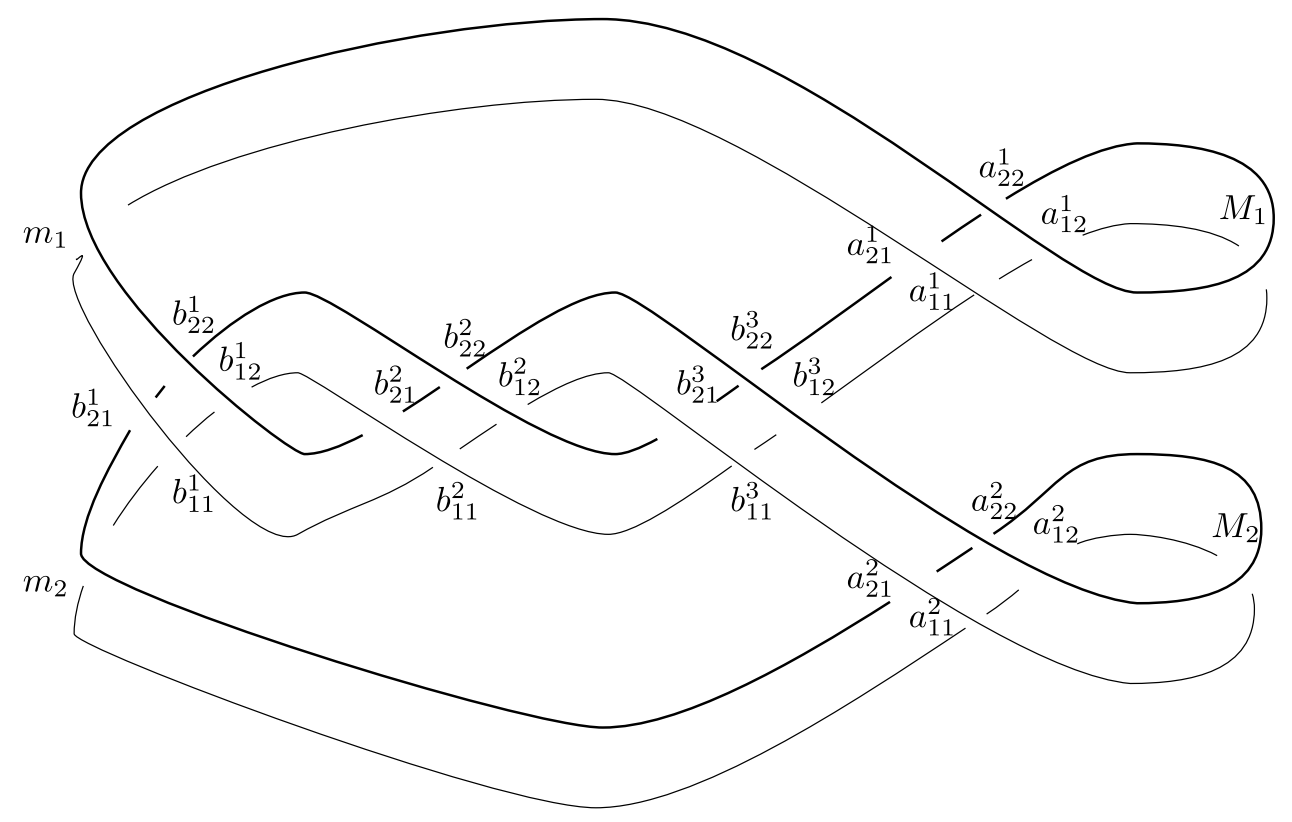

Figure 6. Two copies of the maximal $t b$ trefoil knot. 
where the terms $(\cdots)$ do not involve any continuum chords and are characterized by Theorem 3.2.

This is enough to compute explicitly the long exact sequence. For $\varepsilon_{1}$ and $\varepsilon_{2}$ we get that

$$
\begin{aligned}
& \rho^{\varepsilon_{1}, \varepsilon_{2}}\left(a_{1}\right)=0, \\
& \rho^{\varepsilon_{1}, \varepsilon_{2}}\left(a_{2}\right)=0, \\
& \rho^{\varepsilon_{1}, \varepsilon_{2}}\left(b_{1}\right)=m_{1}+m_{2}, \\
& \rho^{\varepsilon_{1}, \varepsilon_{2}}\left(b_{2}\right)=m_{1}, \\
& \rho^{\varepsilon_{1}, \varepsilon_{2}}\left(b_{3}\right)=m_{1} .
\end{aligned}
$$

In this situation, $\mathrm{LCH}_{0}^{\varepsilon_{1}, \varepsilon_{2}}$ is generated by $\left[b_{2}\right]$. And the map $\rho^{\varepsilon_{1}, \varepsilon_{2}}$ is injective. Similar considerations imply that the map $\sigma: H_{1}\left(S^{1}\right) \rightarrow \mathrm{LCH}_{\varepsilon_{2}, \varepsilon_{1}}^{0}$ is surjective. The map from $\mathrm{LCH}_{\varepsilon_{2}, \varepsilon_{1}}^{0} \rightarrow \mathrm{LCH}_{0}^{\varepsilon_{1}, \varepsilon_{2}}$ is thus 0 .

5.2. Chekanov-Eliashberg knot. Consider the Legendrian knot of Figure 7 .

It is one of the two Legendrian knots which have been distinguished in [5] using Legendrian contact homology. The second one admits only one augmentation and hence has no non-trivial bilinearized Legendrian homology. However, this one admits three augmentations that are (as we will compute) non-equivalent. Again the degree of $a_{i}$ is 1 and the degree of $b_{i}$ is 0 . The

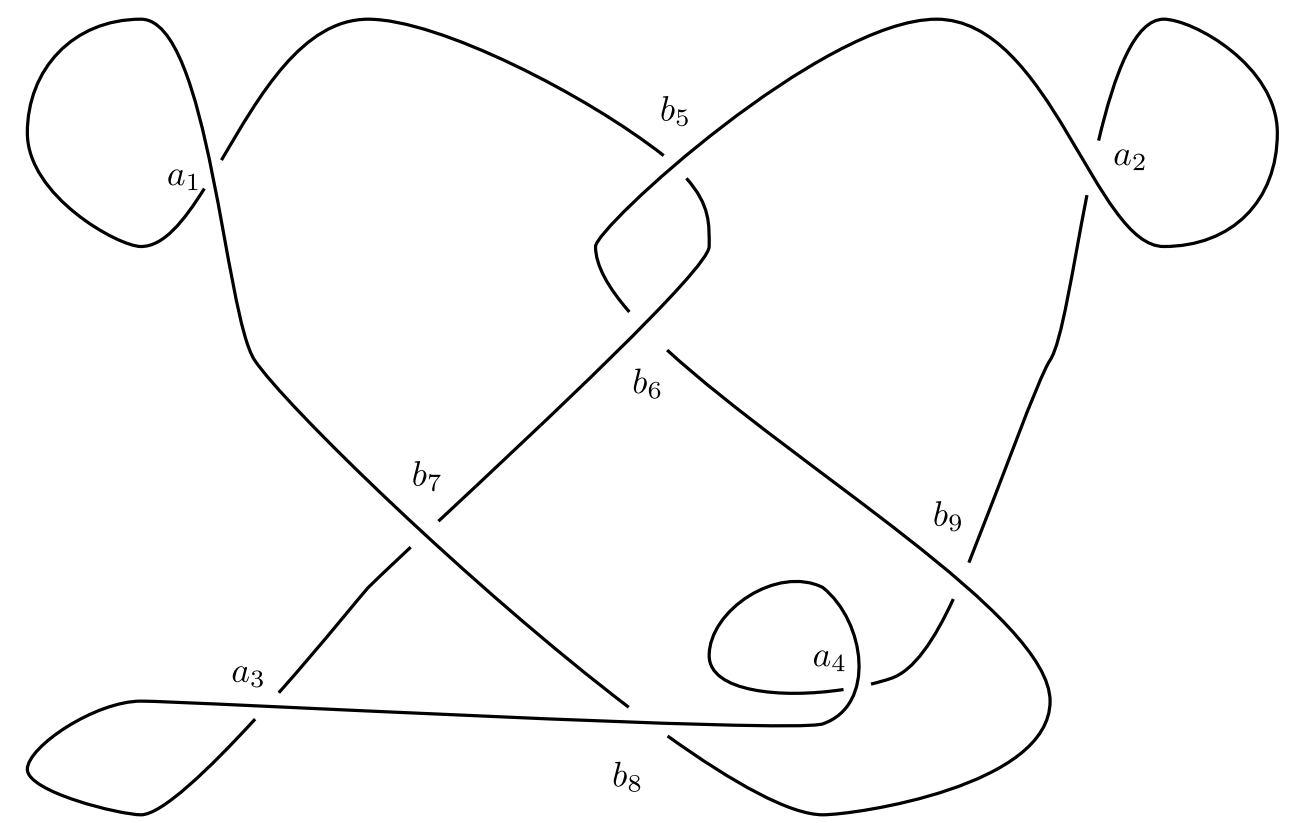

Figure 7. Chekanov-Elisahberg Legendrian knot. 
Table 2. Augmentations of the Chekanov-Eliashberg knot.

\begin{tabular}{cccccc}
\hline & $b_{5}$ & $b_{6}$ & $b_{7}$ & $b_{8}$ & $b_{9}$ \\
\hline$\varepsilon_{1}$ & 0 & 0 & 1 & 1 & 1 \\
$\varepsilon_{2}$ & 0 & 1 & 1 & 1 & 1 \\
$\varepsilon_{3}$ & 1 & 0 & 1 & 1 & 1 \\
\hline
\end{tabular}

differential is given by

$$
\begin{aligned}
& \partial\left(a_{1}\right)=1+b_{7}+b_{7} b_{6} b_{5}+b_{5}+b_{9} b_{8} b_{5}, \\
& \partial\left(a_{2}\right)=1+b_{9}+b_{5} b_{6} b_{9}, \\
& \partial\left(a_{3}\right)=1+b_{8} b_{7} \\
& \partial\left(a_{4}\right)=1+b_{8} b_{9} .
\end{aligned}
$$

The augmentations are listed in Table 2.

For all of these augmentations, the linearized Legendrian contact homologies coincide and are given by $\operatorname{LCH}^{\varepsilon_{i}}(K) \simeq \mathbb{Z}_{2}[1] \oplus \mathbb{Z}_{2}^{2}[0], i=1,2,3$.

However, for any choice of a pair $\varepsilon_{i} \neq \varepsilon_{j}$ of augmentations, we get $\mathrm{LCH}^{\varepsilon_{i}, \varepsilon_{j}}(K) \simeq \mathbb{Z}_{2}[0]$ and we deduce that these three augmentations are pairwise non-equivalent.

As the second Chekanov-Eliashberg example has only one augmentation, we get that the set of equivalence classes of augmentations for those knots are different.

\section{References}

[1] M. Abouzaid and P. Seidel, An open string analogue of Viterbo functoriality. Geom. Topol., 14 (2) (2010), 627-718.

[2] F. Bourgeois, A Morse-Bott approach to contact homology. In Symplectic and contact topology: interactions and perspectives (Toronto, ON/Montreal, QC, 2001), Vol. 35 of Fields Institute Communication, Amer. Math. Soc., Providence, RI, 2003, 55-77.

[3] F. Bourgeois, A survey of contact homology, in 'New perspectives and challenges in symplectic field theory', Vol. 49 of CRM Proc. Lecture Notes, Amer. Math. Soc., Providence, RI, 2009, 45-71.

[4] B. Chantraine, Lagrangian concordance of Legendrian knots, Algebr. Geom. Topol., 10(1) (2010), 63-85.

[5] Y. Chekanov, Differential algebra of Legendrian links, Invent. Math., 150(3) (2002), 441-483.

[6] G. Civan, P. Koprowski, J. Etnyre, J.M. Sabloff and A. Walker, Product structures for Legendrian contact homology, Math. Proc. Cambridge Philos. Soc., 150(2) (2011), 291-311.

[7] T. Ekholm, Rational symplectic field theory over $\mathbb{Z}_{2}$ for exact Lagrangian cobordisms, J. Eur. Math. Soc., 10(3) (2008), 641-704. 
[8] T. Ekholm, Rational SFT, linearized Legendrian contact homology, and Lagrangian Floer cohomology. In 'Perspectives in analysis, geometry, and topology. On the occasion of the 60th Birthday of Oleg Viro', Progr. Math., Birkhäuser/Springer, New York, Vol. 296, Springer, 2012, 109-145.

[9] T. Ekholm, J. Etnyre and M. Sullivan, The contact homology of Legendrian submanifolds in $\mathbb{R}^{2 n+1}$, J. Differ. Geom., 71(2) (2005), 177-305.

[10] T. Ekholm, J. Etnyre and M. Sullivan, Orientations in Legendrian contact homology and exact Lagrangian immersions, Int. J. Math., 16(5) (2005), 453-532.

[11] T. Ekholm, J. Etnyre and M. Sullivan, Legendrian contact homology in $P \times \mathbb{R}$, Trans. Amer. Math. Soc., 359(7) (2007), 3301-3335 (electronic).

[12] T. Ekholm, J.B. Etnyre and J.M. Sabloff, A duality exact sequence for Legendrian contact homology, Duke Math. J., 150(1) (2009), 1-75.

[13] T. Ekholm, K. Honda and T. Kálmán, Legendrian knots and exact Lagrangian cobordisms. preprint, arXiv: 1212.1519, December 2012.

[14] J.B. Etnyre, L.L. Ng and J.M. Sabloff, Invariants of Legendrian knots and coherent orientations. J. Symplectic Geom., 1(2) (2002), 321-367.

[15] D. Fuchs and D. Rutherford, Generating families and Legendrian contact homology in the standard contact space, 2008.

[16] J.M. Sabloff, Duality for Legendrian contact homology, Geom. Topol., 10(2006), 2351-2381 (electronic).

[17] P. Seidel, Fukaya categories and Picard-Lefschetz theory, Zurich Lectures in Advanced Mathematics. European Mathematical Society (EMS), Zürich, 2008.

Laboratoire de Mathématiques d'Orsay, Université Paris-Sud

BLDG. 425

F-91405 Orsay Cedex

FRANCE

E-mail address: bourgeois@math.u-psud.fr

Laboratoire de Mathématiques Jean Leray

BP 92208

2 Rue de la HoussiniéRe

F-44322 Nantes, Cedex 03

FRANCE

E-mail address: baptiste.chantraine@univ-nantes.fr

Received 11/14/2012, accepted 6/14/2013

FB was partially supported by ERC Starting Grant StG-239781-ContactMath. BC was partially supported by the Fonds de la Recherche Scientifique (FRS-FNRS), Belgium. Both of us wish to thank Petya Pushkar for many inspiring conversations without which this project might never have been initiated. The present work is part of the authors activities within CAST, a Research Network Program of the European Science Foundation. 
